\title{
Effect of long-term nutraceutical and dietary supplement use on cognition in the elderly: a 10-year systematic review of randomised controlled trials
}

\author{
Nathan M. D'Cunha ${ }^{1,2}$, Ekavi N. Georgousopoulou ${ }^{1,2,3}$, Lakshika Dadigamuwage ${ }^{1}$, Jane Kellett ${ }^{1,2,3}$, \\ Demosthenes B. Panagiotakos $^{1,3}$, Jackson Thomas ${ }^{1,4}$, Andrew J. McKune ${ }^{2,5,6}$, Duane D. Mellor ${ }^{1,2,4}$ and \\ Nenad Naumovski ${ }^{1,2,4 *}$ \\ ${ }^{1}$ Faculty Health, University of Canberra, Kirinari Street, Bruce, Canberra, ACT 2617, Australia \\ ${ }^{2}$ Collaborative Research in Bioactives and Biomarkers (CRIBB) Group, Kirinari Street, Bruce, Canberra, ACT 2617, Australia \\ ${ }^{3}$ Department of Nutrition-Dietetics, School of Health and Education, Harokopio University, Athens 17671, Greece \\ ${ }^{4}$ University of Canberra Health Research Institute (UC-HRI), University of Canberra, Bruce, Canberra, ACT 2617, Australia \\ ${ }^{5}$ University of Canberra Research Institute for Sport and Exercise (UCRISE), University of Canberra, Bruce, Canberra, ACT \\ 2617, Australia \\ ${ }^{6}$ Discipline of Biokinetics, Exercise and Leisure Science, School of Health Sciences, University of KwaZulu-Natal, Durban, \\ KwaZulu-Natal 4000, South Africa
}

(Submitted 20 June 2017 - Final revision received 24 September 2017 - Accepted 6 November 2017- First published online 9 January 2018)

\section{Abstract}

Nutraceuticals have generated interest as a way to mitigate the cognitive decline in older adults. The aim of this systematic review was to determine the evidence for these claims from the scientific literature in randomised, double-blinded, controlled trials (duration: $\geq 1$ year; participants: $n \geq 100$; age(mean): $\geq 65$ years). Following Preferred Reporting Items for Systematic Reviews and Meta-Analyses (PRISMA) guidelines, we searched four electronic databases (PubMed, Scopus, CINAHL and Web of Science) and identified twenty-five studies published between the 15.June-2006 and 14.June-2016. Interventions included B-vitamins, $n$-3 fatty acids, antioxidant vitamins and herbs. Of the B-vitamin studies, four found benefits to cognition with supplementation. The first of these B-vitamin studies, in individuals with mild cognitive impairment ( $n$ 266; duration $=2$ years $)$, included benefit to executive function $(P=0.015)$ and improvements in the Mini-Mental State Examination (MMSE) among participants with baseline homocysteine above $11.3 \mu \mathrm{mol} / 1(P<0.001)$. In the same sample, the second study found cognitive benefits of B-vitamins dependent on the higher baseline plasma $n$ - 3 fatty acid status. The third B-vitamin study ( $n$ 900; duration $=2$ years) reported improved performance in immediate $(P=0.046)$ and delayed recall $(P=0.013)$, whereas the fourth study $(n 856$; duration $=2$ years) reported slower rate of cognitive decline in the MMSE $(P=0.05)$. One study investigating DHA treatment ( $n$ 402; duration $=1.5$ years) revealed the slower rate of cognitive change in $a p o E$ e 4 non-carriers $(P=0.03)$. As only five included studies revealed notable benefits, presently based on the specific compounds explored here, there is not compelling evidence to support the use nutraceuticals to improve cognition in the elderly. Future long-term trials of nutraceuticals should investigate interactions with lifestyle, blood biomarkers and genetic risk factors.

Key words: Nutraceuticals: Dietary supplements: Cognition: Dementia: Mild cognitive impairment: Elderly: Vitamins

In 2015, the older adult population (over 65 years of age) reached 617 million people worldwide ${ }^{(1)}$, with those aged greater than 80 years being the fastest growing segment of the global population ${ }^{(2)}$. The trend towards an increase in lifespan has led to a rapidly growing interest in the treatment and prevention of diseases associated with ageing, especially neuropsychological conditions, including mild cognitive impairment (MCI) and dementia ${ }^{(3)}$. Pharmacological interventions for these conditions currently provide symptomatic relief, leaving large gaps in the ability to slow dementia disease progression $^{(4)}$ and improve the quality of life (QoL) ${ }^{(5)}$. The worldwide economic burden of dementia is high and increasing, particularly in developing countries, presenting challenges for the public health and aged care systems ${ }^{(2,6)}$. The situation has led to a demand for alternative treatments by individuals, their families and carers attempting to improve the QoL in affected individuals by alleviating the burden of disease $^{(7)}$

Abbreviations: AD, Alzheimer's disease; ADAS-cog, cognitive subscale of Alzheimer's Disease Assessment Scale; APOE4, apoE e4; CDR, Clinical Dementia Rating Scale; CSF, cerebrospinal fluid; FADS, fatty acid desaturase; Hcy, homocysteine; MCI, mild cognitive impairment; MMSE, Mini-Mental State Examination; QoL, quality of life; RCT, randomised controlled trial; TICS, Telephone Interview of Cognitive Status.

*Corresponding author: Dr N. Naumovski, fax +61 26201 5999, email nenad.naumovski@canberra.edu.au 
The theory underpinning the pathophysiology of MCI and dementia include severe neuronal apoptosis, excessive deposition of amyloid plaque, presence of neurofibrillary tangles, atrophy of the hippocampus and impaired brain glucose metabolism $^{(8-10)}$. The consequence of these result in symptoms including decreased cognitive function, memory loss and impaired executive function ${ }^{(11)}$. Increasingly with age, but particularly in those with dementia and MCI, social isolation and poor QoL ensues, with a gradual decrease in the ability to carry out daily functions independently ${ }^{(12)}$. Dementia and MCI are also associated with depressive symptoms and depressive disorders which contribute to a decline in $\mathrm{QOL}^{(13)}$. In addition, the risk of developing these pathologies is increased in carriers of the $a p o E$ e4 (APOE4) allele, an established genetic risk factor for the most common form of dementia, Alzheimer's disease $(\mathrm{AD})^{(14)}$

Recently, it has become more evident that MCI and dementia are predominantly associated with many different factors including the influence of non-modifiable (age, sex and genetics), and modifiable risk (education, physical activity and dietary intake) factors ${ }^{(15)}$. Dietary patterns and intake are of particular interest in the light of promising evidence about the usefulness of the Mediterranean diet in mitigating cognitive decline and dementia ${ }^{(16,17)}$. This evidence has led to increasing scientific interest in evaluating the role of diet and nutrition and starting to view food as a source of consumer-driven management approach of disease ${ }^{(18)}$. Therefore, to boost nutrient intake and increase diet quality, elderly commonly use nutraceuticals and dietary supplements, as a strategy to promote well-being and prevent disease. A recent study ${ }^{(19)}$, identified a sharp rise in the use of nutraceuticals and dietary supplements in the elderly between 2005 and 2011, especially among females. In addition, females are more commonly turning to nutraceutical use, especially those aged over 60 years of age ${ }^{(20)}$. However, questions remain regarding their safety ${ }^{(21)}$, quality ${ }^{(22)}$, potential interactions with conventional medication ${ }^{(23)}$ and their efficacy, that is, the ability to meet certain health claims made by manufacturers ${ }^{(22)}$. An overall lack of regulation of the dietary supplements industry in most jurisdictions poses numerous challenges associated with safety monitoring, particularly due to transactions occurring through the internet ${ }^{(21,22)}$. In addition, certain over the counter supplements may be contaminated by prohibited substances ${ }^{(22)}$, including oestrogenic endocrine disruptors and melamine ${ }^{(24)}$. In the USA, use of dietary supplements is associated with an estimate of more than 23000 emergency room visits per year ${ }^{(25)}$. Numerous nutraceuticals, including multivitamins and iron, are associated with higher risk of total mortality in elderly females ${ }^{(26)}$. Despite this, the dietary supplements industry sales continue to grow with a thriving niche market for products that claim to improve cognitive function and promote brain health.

Typically nutraceuticals and dietary supplements are concentrated bioactive substances derived from foods or herbs and are usually, but not exclusively, consumed in a pill or powder form ${ }^{(27)}$. The term 'nutraceuticals' commonly includes drugs, food ingredients and dietary supplements generally without patent protection ${ }^{(28)}$. For this review, 'nutraceuticals' are defined as non-patented products and substances that do not contribute to overall energy intake but provide a potential physiological benefit or protection against chronic disease ${ }^{(27,28)}$. Although often used interchangeably with nutraceuticals, not all dietary supplements are sold with claims of protection against chronic disease. Therefore, this review included only dietary supplements with nutraceutical properties. The term nutraceutical and functional foods have also been used interchangeably ${ }^{(29)}$, and it should be noted that functional foods may also include fortified food products; such as margarine with added $n-3$ fatty acids ${ }^{(30)}$. The main categories of nutraceuticals and dietary supplements are vitamins, minerals, herbal medicines, probiotics, $n$ - 3 PUFA, antioxidants and polyphenol supplements. Some of the claims include memory enhancement ${ }^{(31)}$, maintenance of memory function ${ }^{(31)}$, and protection from cell degeneration ${ }^{(32)}$, whereas others suggest that nutraceuticals exhibit beneficial outcomes in certain conditions such as $\mathrm{AD}^{(33)}$. Nevertheless, the perception of efficacy in relation to the scientific literature available requires a systematic analysis and assessment.

This systematic review aims to analyse the recent evidence concerning the use of nutraceuticals and dietary supplements over the last 10 years and their associated effects on cognition in elderly individuals in recent, large randomised, double-blinded, placebo-controlled, human trials of at least 1-year duration and with large participant cohorts.

\section{Methods}

A systematic review of published literature was performed to identify the evidence for the long-term use of common nutraceuticals and dietary supplements to improve cognition in elderly participants (aged $\geq 65$ years).

\section{Search strategy}

Following Preferred Reporting Items for Systematic Reviews and Meta-Analyses (PRISMA) 2009 guidelines ${ }^{(34)}$, literature searches were conducted across four electronic databases (PubMed, Scopus, CINAHL and Web of Science), to identify the most recent studies published in the last 10 years. The study was established on the 15 June 2016, therefore only articles published from 15 June 2006 to 14 June 2016 were eligible for inclusion. The search strategy was re-run on the 2 September 2017 , and one further article from within our date range was located for inclusion into the systematic review. The popularity of dietary supplements has increased since 2005 and $2006^{(19)}$, whereas evidence surrounding the long-term use of nutraceuticals and dietary supplements has strengthened since 2006, in particular in relation to the evidence surrounding B-vitamins and cognitive function ${ }^{(35,36)}$. Observational studies are limited by heterogeneity, a reliance on self-reported nutritional data and confounding due to socioeconomic factors ${ }^{(17)}$. Therefore, to attempt to avoid such limitations and to best examine the potential presence of causality, only randomised, double-blind, placebo-controlled human trials examining the effects of nutraceuticals and dietary supplements on cognition in older adults were identified. The database search used keywords relating to the aim of the paper; that is 'Alzheimer*', 'dementia' or 'cognitive impairment'; 'supplement"' or 'nutraceutical*', and 
'trial*'; and 'control*' or 'random*'. Where applicable, the search option 'clinical trial' or 'controlled trial' was selected to narrow the search results before the screening. During the search, the reference lists of other review articles were also manually searched $^{(35,37)}$

\section{Eligibility criteria}

To best determine the efficacy of nutraceuticals and dietary supplements, only human, double-blinded, randomised, placebo-controlled studies $>1$ year in duration, with participant cohorts consisting of 100 or more elderly human subjects aged over 65 years, were eligible for inclusion. The duration of 1 year was considered essential as a minimal time period for nutraceutical interventions to potentially influence cognitive function $^{(38)}$. Studies published in peer-reviewed journals only in English language were selected for the title and abstract screening. Studies were included if the primary or secondary outcomes pertained to cognitive function, cognitive performance, cognitive impairment, QoL, brain atrophy and the prevention of cognitive decline. A range of outcomes were included such as: Screening measures for cognitive impairment (i.e. Mini-Mental State Examination (MMSE)); cognitive batteries (i.e. cognitive subscale of Alzheimer's Disease Assessment Scale (ADAS-cog)); and tests of cognitive function (i.e. Rey Auditory Verbal Learning Test (RAVLT) and California Verbal Learning Test (CVLT)). If the eligibility criteria could be met or not be determined based on the information provided in the title and abstract, then the article was retrieved and screened for inclusion.

\section{Selection criteria and quality assessment}

The reasons for exclusion of full-text articles included not sustaining a placebo arm for the full duration of the study, a mean age of $<65$ years and no measurement of cognitive outcomes. Articles testing the effects of medications in combination with a nutraceutical were excluded due to the inability to distinguish the beneficial properties of the nutraceutical per se. The included studies contained measures of cognitive function or screening measures as either a primary or secondary outcome. Although some studies included additional measures for depression, mood and behavioural difficulties, these results were not of interest to this review. The flow chart describing the process of the study selection is represented in Fig. 1. All full-text articles were reviewed and independently assessed for risk of bias by two reviewers (N. M. D. C. and E. N. G.) (Table 5) using the criteria suggested in the Cochrane guidelines $^{(39)}$.

\section{Results}

\section{Description of studies}

A total of 1489 records were identified from initial electronic database searches, and five were identified through hand searching. In all, twenty-five studies were included following both title and abstract screening and subsequent assessment of the full-text. For this review, articles were categorised into four categories:

(1) products containing more than one type of nutraceutical or dietary supplements (Table 1);

(2) B-vitamins (products containing B-vitamins only or individual B-vitamins) (Table 2);

(3) $n$-3 fatty acids (or a combination of) (Table 3); and

(4) nutraceuticals or dietary supplements that did not fall within the first three categories (Table 4).

\section{Outcome measures}

These main outcome measures included but were not limited to:

(1) MMSE score: 30-point questionnaire commonly used to measure cognitive impairment;

(2) Telephone Interview of Cognitive Status (TICS): global mental status test administered over the telephone;

(3) ADAS-cog: most frequently used test to measure cognition in clinical trials, consisting of eleven memory, language, and attention tasks;

(4) RAVLT: common test for episodic declarative memory;

(5) The Clinical Dementia Rating Scale (CDR): used to quantify the severity of symptoms of dementia (i.e. its 'stage');

(6) CVLT: commonly used to measure episodic verbal memory;

(7) Alzheimer's Disease Cooperative Study-activities of daily living inventory: used for the assessment of severe cases of $\mathrm{AD}$; and

(8) East Boston Memory Test (EBMT): short story recollection task.

The MMSE (or equivalent variant) was the most commonly used, which was applied in eighteen studies $^{(11,30,36,37,43,44,46-49,52-55,57-60)}$; whereas the TICS or TICS-modified (TICS-m) was used in nine studies $^{(36,37,40,41,46,50,53,54,56)}$ and the ADAS-cog was used in six studies $^{(37,44,52-54,58)}$, respectively.

\section{Effect of nutraceuticals and dietary supplements on cognitive function in elderly individuals}

Combinations of different nutraceuticals. Two included studies $^{(41,42)}$ tested the implementation of a broad-spectrum, once daily, multivitamin supplement on cognitive function in elderly individuals. The 12-year long study by Grodstein et al. ${ }^{(41)}$ was a subgroup of the Physicians' Health Study II, which enrolled 5947 male physicians (age: 71.6 years and supplied them with Centrum ${ }^{\circledR}$ Silver, a commercially available multivitamin (composition in Table 1). This highly educated, health-conscious cohort reported no benefits in overall cognitive performance over three follow-up visits (mean duration between assessments: 2 years between 1st and 2nd, 4 years between 2nd and 3rd, 4 years between 3 rd and 4th) based on a composite score including TICS and EBMT (both, $P>0.05$ ). In the global composite score, the difference in cognitive change between placebo and multivitamin groups over the follow-up 


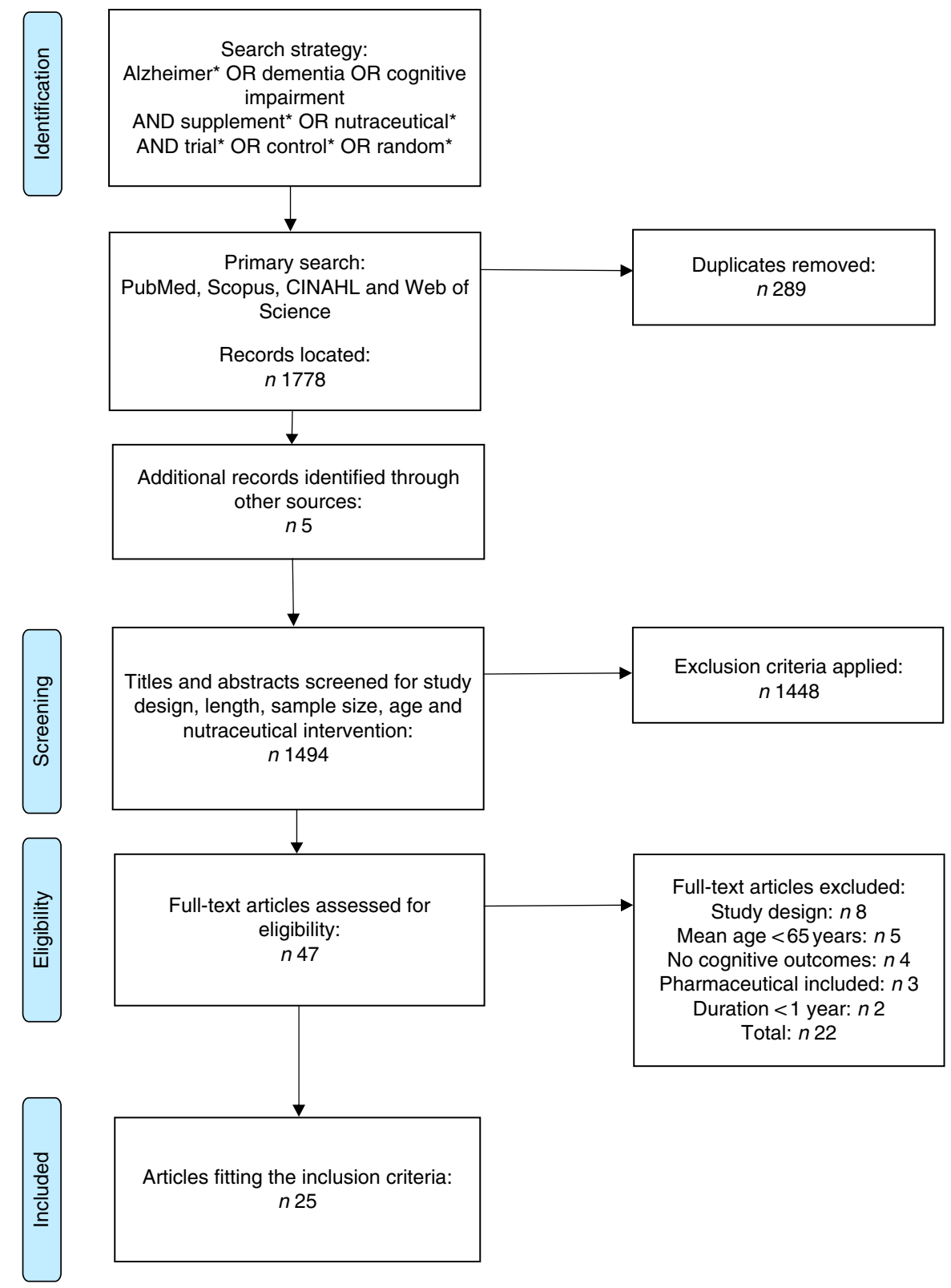

Fig. 1. Preferred Reporting Items for Systematic Reviews and Meta-Analyses (PRISMA) flow chart summary of systematic review search process.

period was -0.01 statistical unit (SU). To our knowledge, to date, this is the longest study investigating the effect of a nutraceutical on cognition. However, there are numerous limitations found with this study including selection bias. Male physicians by their vocation are required to have excellent cognition and may already have been consuming a healthy diet and thus derived little to no benefit from additional vitamins and minerals. They are also wealthier and rarely smoke, drink or use recreational drugs. A large proportion of the participants may have also benefited from previous successful long-term treatment with aspirin in the Physicians' Health Study I ${ }^{(61)}$. It is also possible that higher doses of certain vitamins with may be necessary to elicit the desired effect, whereas multivitamin absorption and bioavailability has been questioned ${ }^{(62)}$. It was also reported that the participants had 83.5 and $84.2 \%$ compliance at least two-thirds of the time, which were likely to influence the results. However, a trial published in 2007 using a multivitamin or placebo, given to 910 participants including 431 females, also reported no benefits on cognitive function in older participants $^{(42)}$. In addition, no benefit for digit span forward and verbal fluency scores was found over 1 year. Further analysis suggested a weak benefit in verbal fluency scores for participants aged over 75 years (mean difference of $2.8 \mathrm{SU}$, $\mathrm{p}$ for the difference between groups $>0.05$ ) who were also at risk of micronutrient deficiency based upon a simple 17 item questionnaire at baseline. Compared with the 12-year study in physicians $^{(41)}$, this study ${ }^{(42)}$ had lower compliance (78\%). However, it is likely that participants experiencing a decline in cognition may have been lost to follow-up. In addition, only short-term memory and executive function were assessed. 
Table 1. The effect of combinations of nutraceuticals and dietary supplements on cognition in elderly participants (Mean values and standard deviations; medians and interquartile ranges (IQR))

\begin{tabular}{|c|c|c|c|c|c|c|c|c|}
\hline $\begin{array}{l}\text { First } \\
\text { author }\end{array}$ & Nutraceutical & Country & Design & Relevant selection criteria & Subjects & Intervention & Cognitive outcomes & Results \\
\hline $\begin{array}{l}\text { Chew } \\
\quad \text { et al. }{ }^{(40)}\end{array}$ & $\begin{array}{l}n-3 \text { FA, } \\
\quad \text { Lutein and } \\
\text { zeaxanthin }\end{array}$ & USA & $\begin{array}{l}2 \times 2 \text { factorial, } \\
\text { double-blind, } \\
\text { placebo- } \\
\text { controlled RCT }\end{array}$ & $\begin{array}{l}\text { Inclusion criteria: individuals at } \\
\text { high risk of AMD } \\
\text { Exclusion: previous ocular } \\
\text { disease or retinal surgery } \\
\text { procedure, previous } \\
\text { supplementation with } 2 \mathrm{mg} \text { or } \\
\text { more of lutein and } / \text { or } 500 \mathrm{mg} \text { of } \\
n-3 \text { for } 1 \text { year } \\
\text { or more }\end{array}$ & $\begin{array}{l}n 3741(\mathrm{~F}=1767 \\
\text { and } \mathrm{M}=1306) \\
\text { Age }=72 \cdot 7 \\
\text { (sD 7.8) years } \\
\text { (placebo), } 72 \cdot 7 \\
\text { (sD 7.7) years } \\
\text { ( } n-3 \text { group) }\end{array}$ & $\begin{array}{l}\text { Duration }=5 \text { years } \\
\text { Four arms: (1) placebo, (2) } 350 \mathrm{mg} \mathrm{DHA} / \\
650 \mathrm{mg} \text { EPA, (3) } 10 \mathrm{mg} \text { lutein/ } 2 \mathrm{mg} \\
\text { zeaxanthin, (4) both } 3 \text { and } 4 \text { (all daily) }\end{array}$ & $\begin{array}{l}\text { Primary = yearly change in composite } \\
\text { scores of: TICS-m, verbal fluency, letter } \\
\text { fluency, alternating fluency, WAIS } \\
\text { (logical memory I and II - immediate } \\
\text { and delayed recall), digit backwards, } \\
\text { delayed recall of WMS-III recall) }\end{array}$ & $\begin{array}{l}\text { There was no statistically } \\
\text { significant effect on } \\
\text { cognitive function in this } \\
\text { group of individuals with } \\
\text { intermediate AMD or } \\
\text { late AMD (all } P>0.05 \text { ) }\end{array}$ \\
\hline $\begin{array}{l}\text { Grodstein } \\
\text { et al. }{ }^{(41)}\end{array}$ & Multivitamin & USA & $\begin{array}{l}2 \times 2 \times 2 \times 2 \\
\text { factorial double- } \\
\text { blind, placebo- } \\
\text { controlled RCT }\end{array}$ & $\begin{array}{l}\text { Inclusion criteria: healthy male } \\
\text { physicians } \\
\text { Exclusion criteria: concurrent } \\
\text { vitamin and/or multivitamin } \\
\text { supplementation }\end{array}$ & $\begin{array}{l}n 5947(\mathrm{M}) \\
\text { Age }=71 \cdot 6 \\
\quad \text { (sD 5.9) years } \\
\text { (placebo), 71.6 } \\
\text { (sD 6.0) years } \\
\text { (multivitamin } \\
\text { group) }\end{array}$ & $\begin{array}{l}\text { Duration }=12 \text { years } \\
\text { Placebo or multivitamin (Centrum Silver) } \\
\text { (daily) }\end{array}$ & $\begin{array}{l}\text { Primary }=\text { composite score (average) of: } \\
\text { TICS, EBMT, intermediate and delayed } \\
\text { recall of a 10-word list and category } \\
\text { fluency }\end{array}$ & $\begin{array}{l}\text { The overall cognitive } \\
\text { performance was not } \\
\text { significantly different } \\
\text { between the } \\
\text { multivitamin group and } \\
\text { placebo group } \\
(P=0.65)\end{array}$ \\
\hline $\begin{array}{l}\text { McNeill } \\
\text { et al. }{ }^{(42)}\end{array}$ & $\begin{array}{l}\text { Multivitamin/ } \\
\text { multimineral }\end{array}$ & UK & $\begin{array}{l}\text { Double-blind, } \\
\text { placebo- } \\
\text { controlled RCT }\end{array}$ & $\begin{array}{l}\text { Exclusion criteria: individuals with } \\
\text { dementia, individuals taking } \\
\text { vitamins, minerals or fish oils } \\
\text { within } 3 \text { months of recruitment } \\
(1 \text { month for water-soluble } \\
\left.\text { vitamins other than } B_{12}\right) \text {. }\end{array}$ & $\begin{array}{l}n 910(\mathrm{~F}=431 \text { and } \\
\mathrm{M}=479) \\
\text { Age }=71 \text { years } \\
(1 \mathrm{st}-3 \mathrm{rd} \text { IQR } \\
68 \cdot 0-76 \cdot 0) \\
\text { (placebo), } 72 \\
\text { years }(1 \mathrm{st}-3 \mathrm{rd} \\
\text { IQR 68.0-76.0) } \\
\text { (supplement } \\
\text { group) }\end{array}$ & $\begin{array}{l}\text { Duration }=1 \text { year } \\
\text { Placebo or treatment consisting of one tablet } \\
\text { per d containing: } 800 \mu \mathrm{g} \text { vitamin } \mathrm{A}, 60 \mathrm{mg} \\
\text { vitamin } \mathrm{C}, 5 \mu \mathrm{g} \text { vitamin } \mathrm{D}, 10 \mathrm{mg} \text { vitamin } \mathrm{E}, \\
1.4 \mathrm{mg} \text { thiamin, } 1.6 \mathrm{mg} \text { riboflavin, } 18 \mathrm{mg} \\
\text { niacin, } 6 \mathrm{mg} \text { pantothenic acid, } 2 \mathrm{mg} \\
\text { pyridoxine, } 1 \mu \mathrm{g} \text { vitamin } \mathrm{B}_{12}, 200 \mu \mathrm{g} \text { folic } \\
\text { acid, } 14 \mathrm{mg} \mathrm{Fe}, 150 \mu \mathrm{g} \text { iodine, } 0.75 \mathrm{mg} \mathrm{Cu} \text {, } \\
15 \mathrm{mg} \mathrm{Zn} \text {, and } 1 \mathrm{mg} \mathrm{Mn}\end{array}$ & $\begin{array}{l}\text { Primary }=\text { Digit span forward score and } \\
\text { verbal fluency score }\end{array}$ & $\begin{array}{l}\text { No significant change in } \\
\text { either test between the } \\
\text { placebo and } \\
\text { supplement group } \\
\text { (all } P>0.05 \text { ) }\end{array}$ \\
\hline $\begin{array}{l}\text { Naeini } \\
\quad \text { et al. }{ }^{(11)}\end{array}$ & $\begin{array}{l}\text { Vitamin } C \text { and } \\
\text { vitamin } E\end{array}$ & Iran & $\begin{array}{l}\text { Double-blind, } \\
\text { placebo- } \\
\text { controlled RCT }\end{array}$ & $\begin{array}{l}\text { Inclusion criteria: MMSE score } \\
\text { (Iranian version) between } 21 \\
\text { and } 26 \\
\text { Exclusion criteria: disabling } \\
\text { disease, alcohol intake, } \\
\text { smoking, routine consumption } \\
\text { of neurological or antioxidant } \\
\text { drugs }\end{array}$ & $\begin{array}{l}n=256(F=136 \\
\text { and } M=120) \\
\text { Age }=66.3 \\
\text { (sD } 0.38 \text { ) years } \\
\text { (placebo), 66.5 } \\
\text { (sD } 0.39 \text { ) years } \\
\text { (supplement) }\end{array}$ & $\begin{array}{l}\text { Duration }=1 \text { year } \\
\text { Placebo or } 300 \mathrm{mg} \text { of vitamin } \mathrm{E}(\mathrm{DI}- \\
\quad \text {-tocopherol acetate) plus } 400 \mathrm{mg} \text { of } \\
\text { vitamin C (ascorbic acid) (daily) }\end{array}$ & Primary $=$ MMSE & $\begin{array}{l}\text { No beneficial effect of } \\
\text { vitamin } E \text { and vitamin } C \\
\text { were observed on } \\
\text { cognitive function as } \\
\text { measured by the MMSE } \\
(P=0.88)\end{array}$ \\
\hline $\begin{array}{l}\text { Rossom } \\
\text { et al. }{ }^{(43)}\end{array}$ & $\begin{array}{l}\text { Ca and vitamin } \\
D_{3}\end{array}$ & USA & $\begin{array}{l}\text { Double-blind, } \\
\text { placebo- } \\
\text { controlled RCT }\end{array}$ & $\begin{array}{l}\text { Inclusion criteria: no evidence of a } \\
\text { medical condition } \\
\text { Exclusion criteria: } \\
\text { hypercalcaemia, renal calculi, } \\
\text { corticosteroid use, calcitriol } \\
\text { use }\end{array}$ & $\begin{array}{l}n 2034(\mathrm{~F}) \\
\text { Age }=70 \cdot 9 \text { years } \\
\text { (placebo), } 70 \cdot 7 \\
\text { years } \\
\text { (Ca and vitamin } \\
\text { D group) }\end{array}$ & $\begin{array}{l}\text { Duration }=7 \text { years } \\
\text { Placebo or treatment consisted of } 1 \mathrm{~g} \text { of } \\
\text { calcium carbonate and } 400 \mathrm{IU}(10 \mathrm{\mu g}) \text { of } \\
\text { vitamin } D_{3} \text { per } d\end{array}$ & $\begin{array}{l}\text { Primary = classification of probable } \\
\text { dementia, MCI, or cognitively normal } \\
\text { Secondary = MMSE, WHISCA cognitive } \\
\text { battery (Digit Span forward and } \\
\text { backwards test, primary mental abilities } \\
\text { vocabulary test, test for spatial ability, } \\
\text { verbal fluency tests, CVLT, BVRT and } \\
\text { finger tapping test) }\end{array}$ & $\begin{array}{l}\text { No significant association } \\
\text { between treatment, } \\
\text { incident dementia, } \mathrm{MCl} \\
\text { or cognitive function } \\
\text { (all } P>0.05 \text { ) }\end{array}$ \\
\hline
\end{tabular}

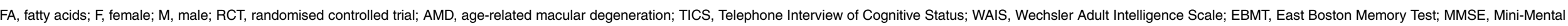
State Examination; MCl, mild cognitive impairment; WHISCA, Women's Health Initiative Study of Cognitive Aging; CVLT, California Verbal Learning Test; BVRT, Benton Visual Retention Test; WMS, Wechsler Memory Scale. 


\section{N's British Journal of Nutrition}

Table 2. The effect of B-vitamins on cognition in elderly participants (Mean values and standard deviations)

\begin{tabular}{|c|c|c|c|c|c|c|c|c|}
\hline First author & Nutraceutical & Country & Design & Relevant selection criteria & Subjects & Intervention & Cognitive outcomes & Results \\
\hline Aisen et al. ${ }^{(44)}$ & $\begin{array}{l}\text { Folic acid, vitamin } B_{6} \text { and } \\
\text { vitamin } B_{12}\end{array}$ & USA & $\begin{array}{l}\text { Parallel-group, } \\
\text { double-blind, } \\
\text { placebo- } \\
\text { controlled RCT }\end{array}$ & $\begin{array}{l}\text { Inclusion criteria: MMSE within } 14 \\
\text { and } 26 \\
\text { Exclusion criteria: vitamin } \\
B_{12}<175 \mathrm{pg} / \mathrm{ml} \text {, folate }<4.2 \mathrm{ng} / \mathrm{ml} \text {, } \\
\text { serum creatinine } \geq 0.02 \mathrm{~g} / \mathrm{l}\end{array}$ & $\begin{array}{l}n 409 \\
\text { Age }=77 \cdot 3 \text { (sD 7.9) years } \\
\text { (placebo), } 75 \cdot 7 \\
\text { (sD 8.0) years } \\
\text { (B-vitamin group) }\end{array}$ & $\begin{array}{l}\text { Duration }=1.5 \text { years } \\
\text { Placebo or treatment } \\
\text { consisting of } 5 \mathrm{mg} \text { folic } \\
\text { acid, } 1 \mathrm{mg} \text { vitamin } B_{12} \\
\text { and } 25 \mathrm{mg} \text { of vitamin } B_{6} \\
\text { per d }\end{array}$ & $\begin{array}{l}\text { Primary }=\text { ADAS-cog. } \\
\text { Secondary }=\text { MMSE, } \\
\text { CDR, ADCS-ADL } \\
\text { and QOL-AD }\end{array}$ & $\begin{array}{l}\text { Despite an increase in B-vitamin } \\
\text { levels and reduced Hcy, there were } \\
\text { no cognitive improvements } \\
\text { compared with placebo }(P=0.52)\end{array}$ \\
\hline Dangour et al. ${ }^{(45)}$ & Vitamin $B_{12}$ & UK & $\begin{array}{l}\text { Double-blind, } \\
\text { placebo- } \\
\text { controlled RCT }\end{array}$ & $\begin{array}{l}\text { Inclusion criteria: } \geq 24 \\
\text { Exclusion criteria: current vitamin } \\
\mathrm{B}_{12} \text { consumption, vitamin } \mathrm{B}_{12} \\
\text { injection, vitamin } \\
\mathrm{B}_{12}<107 \mathrm{pmol} / \mathrm{ll}, \mathrm{Hb}<110 \mathrm{~g} / \mathrm{l} \\
\text { (F) and }<120 \mathrm{~g} / \mathrm{l}(\mathrm{M})\end{array}$ & $\begin{array}{l}n 201(\mathrm{~F}=107 \text { and } \\
\mathrm{M}=94) \\
\text { Age }=80 \cdot 1 \text { (sD } 3 \cdot 7) \text { years } \\
\text { (placebo), } 79 \cdot 9 \text { (sD } \\
\text { 3.5) years (B-vitamin } \\
\text { group) }\end{array}$ & $\begin{array}{l}\text { Duration }=1 \text { year } \\
\text { Placebo or } 1 \mathrm{mg} \text { vitamin } \\
\mathrm{B}_{12} \text { (daily) }\end{array}$ & $\begin{array}{l}\text { Primary }=\text { CVLT. } \\
\text { Secondary }=\text { oral } \\
\text { symbol letter } \\
\text { modality test, } \\
\text { simple and choice } \\
\text { reaction time, } \\
\text { verbal fluency test }\end{array}$ & $\begin{array}{l}\text { No evidence of an effect on cognitive } \\
\text { function from the treatment } \\
\text { (all } P>0.05 \text { ) }\end{array}$ \\
\hline de Jager et al. ${ }^{(46)}$ & $\begin{array}{l}\text { Folic acid, vitamin } B_{6} \text { and } \\
\text { vitamin } B_{12}\end{array}$ & UK & $\begin{array}{l}\text { Double-blind, } \\
\text { placebo- } \\
\text { controlled RCT }\end{array}$ & $\begin{array}{l}\text { Inclusion criteria: MCI determined } \\
\text { by TICS-m } \geq 17 \text { and } \leq 29 \text { out of } \\
39 \text {, category fluency test, } \\
\text { MMSE } \geq 24 \text {, CAMDEX, ADL }\end{array}$ & $\begin{array}{l}n 266(\mathrm{~F}=163 \text { and } \\
\mathrm{M}=60) \\
\text { Age }=76 \cdot 7 \text { (sD } 4.8) \text { years } \\
\text { (placebo), } 76 \cdot 8 \text { (sD } \\
5 \cdot 1) \text { years (B-vitamin } \\
\text { group) }\end{array}$ & $\begin{array}{l}\text { Duration }=2 \text { years } \\
\text { Placebo or } 0.8 \mathrm{mg} \text { folic } \\
\text { acid, } 20 \mathrm{mg} \text { vitamin } \mathrm{B}_{6} \\
\text { and } 0.5 \mathrm{mg} \text { vitamin } B_{12} \\
\text { (daily) }\end{array}$ & $\begin{array}{l}\text { Secondary = MMSE, } \\
\text { HVLT-R, sematic } \\
\text { memory } \\
\text { (CERAD), CDR, } \\
\text { CLOX, IQCODE }\end{array}$ & $\begin{array}{l}\text { Hcy decreased in the B-vitamin group } \\
\text { but not the placebo group } \\
(P<0.001) \text {. No improvements were } \\
\text { observed for MMSE, HVLT-R or } \\
\text { CERAD. However, the CLOX test of } \\
\text { executive function showed } \\
\text { significant improvement relative to } \\
\text { placebo }(P=0.015) \text {. Subgroup } \\
\text { analysis (baseline Hcy }>11.3 \mu \text { mol/ } \\
\text { I) found benefit to B-vitamins in the } \\
\text { MMSE }(P<0.001) \text {, HVLT-R } \\
(P=0.001) \text { and semantic memory } \\
(P=0.037)\end{array}$ \\
\hline Ford et al. ${ }^{(37)}$ & $\begin{array}{l}\text { Folic acid, vitamin } B_{6} \text { and } \\
\text { vitamin } B_{12}\end{array}$ & Australia & $\begin{array}{l}\text { Double-blind, } \\
\text { placebo- } \\
\text { controlled RCT }\end{array}$ & $\begin{array}{l}\text { Inclusion criteria: males from } \\
\text { abdominal aortic screening } \\
\text { study } \\
\text { Exclusion criteria: BDI score } \geq 18 \\
\text { or MMSE } \leq 24 \text {, live in residential } \\
\text { care setting, taking B-vitamin } \\
\text { supplements }\end{array}$ & $\begin{array}{l}n 299(\mathrm{M}) \\
\text { Age }=78 \cdot 7 \text { (sD 2.7) years } \\
\text { (placebo), } 79 \cdot 3 \text { (sD } \\
\text { 2.8) years (B-vitamin } \\
\text { group) }\end{array}$ & $\begin{array}{l}\text { Duration }=2 \text { years } \\
\text { Placebo or } 2 \mathrm{mg} \text { folic acid, } \\
25 \mathrm{mg} \text { vitamin } \mathrm{B}_{6} 400 \mu \mathrm{g} \\
\text { vitamin } \mathrm{B}_{12} \text { (daily) }\end{array}$ & $\begin{array}{l}\text { Primary }=\text { ADAS-cog } \\
\text { Secondary }=\text { CVLT, } \\
\text { MMSE, Digit } \\
\text { cancellation test, } \\
\text { CLOX and TICS }\end{array}$ & $\begin{array}{l}\text { Compared with placebo, no significant } \\
\text { improvement was observed with } \\
\text { cognitive function in elderly men } \\
(P=0.478)\end{array}$ \\
\hline Kwok et al. ${ }^{(47)}$ & $\begin{array}{l}\text { Folic acid and vitamin } B_{12} \\
\text { (methylcobalamin) }\end{array}$ & Hong Kong & $\begin{array}{l}\text { Double-blind, } \\
\text { placebo- } \\
\text { controlled RCT }\end{array}$ & $\begin{array}{l}\text { Inclusion criteria: diagnosed with } \\
\text { dementia } \\
\text { Exclusion criteria: vitamin } \\
\mathrm{B}_{12}<150 \mathrm{pmol} / \mathrm{I} \\
\text { folate }<9.5 \mathrm{nmol} / / \\
\text { creatinine }>250 \mu \mathrm{mol} / \mathrm{l} \\
\text { hypothyroidism }\end{array}$ & $\begin{array}{l}n 140(\mathrm{~F}=89 \text { and } \\
\mathrm{M}=51) \\
\text { Age }=77 \cdot 2(\mathrm{sD} 7.9) \text { years } \\
\text { (placebo), } 79 \cdot 1 \text { (sD } \\
6.7) \text { years (B-vitamin } \\
\text { group) }\end{array}$ & $\begin{array}{l}\text { Duration }=18 \text { months } \\
\text { Placebo or } 5 \mathrm{mg} \text { folic acid } \\
\text { and } 1 \mathrm{mg} \text { of } \\
\text { methylcobalamin (daily) }\end{array}$ & $\begin{array}{l}\text { Primary = battery } \\
\text { consisting of } \\
\text { MMSE and } \\
\text { Chinese Mattis } \\
\text { dementia rating } \\
\text { scale }\end{array}$ & $\begin{array}{l}\text { No significant differences between } \\
\text { groups }\end{array}$ \\
\hline McMahon et al. ${ }^{(48)}$ & $\begin{array}{l}\text { Folate (I-5- } \\
\text { methyltetrahydrofolate), } \\
\text { vitamin } \mathrm{B}_{6} \text { (pyridoxine) and } \\
\text { vitamin } \mathrm{B}_{12} \text { (cobalamin) }\end{array}$ & New Zealand & $\begin{array}{l}\text { Double-blind, } \\
\text { placebo- } \\
\text { controlled RCT }\end{array}$ & $\begin{array}{l}\text { Inclusion criteria: fasting } \\
\text { Hcy } \geq 13 \mu \mathrm{mol} / /, \text { plasma } \\
\text { creatinine } \leq 133 \mu \mathrm{mol} / \mathrm{I}(\mathrm{W}) \text { and } \\
\leq 115 \mu \mathrm{mol} / /(\mathrm{M}) \\
\text { Exclusion criteria: suspected } \\
\text { dementia (MMSE), taking } \\
\text { vitamin supplements (folic acid, } \\
\text { vitamin } \mathrm{B}_{12} \text { or } \mathrm{B}_{6} \text { ), receiving } \\
\text { treatment for depression, } \\
\text { diabetes, history of stroke or } \\
\text { transient ischaemic attacks }\end{array}$ & $\begin{array}{l}n 276(F=112 \text { and } \\
M=156) \\
\text { Age }=73 \cdot 4(\text { sD } 5 \cdot 7) \text { years } \\
\text { (placebo), } 73 \cdot 6 \text { (sD } \\
5 \cdot 8) \text { years (B-vitamin } \\
\text { group) }\end{array}$ & $\begin{array}{l}\text { Duration }=2 \text { years } \\
\text { Placebo or } 1000 \mu \mathrm{g} \\
\text { folate (I-5- } \\
\text { methyltetrahydrofolate), } \\
10 \mathrm{mg} \text { vitamin } \mathrm{B}_{6} \text { and } \mu \mathrm{g} \\
\text { vitamin } \mathrm{B}_{12} \text { (cobalamin) }\end{array}$ & $\begin{array}{l}\text { Primary }=\text { MMSE, } \\
\text { RAVLT, Controlled } \\
\text { Oral Word } \\
\text { Association Test } \\
\text { of the Multilingual } \\
\text { Aphasia } \\
\text { Examination, } \\
\text { Paragraph recall } \\
\text { from Weschler } \\
\text { memory scales, } \\
\text { Category word } \\
\text { fluency test, } \\
\text { Reitan Trail } \\
\text { Making Test and } \\
\text { Raven's } \\
\text { Progressive } \\
\text { Matrices test }\end{array}$ & $\begin{array}{l}\text { Despite lowering Hcy, raising plasma } \\
\text { folate and plasma vitamin } B_{12} \text {, the } \\
\text { intervention did not improve } \\
\text { cognitive function (all } P>0.05 \text { ). }\end{array}$ \\
\hline
\end{tabular}




\section{NS British Journal of Nutrition}

Table 2. Continued

\begin{tabular}{|c|c|c|c|c|c|c|c|c|}
\hline First author & Nutraceutical & Country & Design & Relevant selection criteria & Subjects & Intervention & Cognitive outcomes & Results \\
\hline Oulhaj et al. ${ }^{(36)}$ & $\begin{array}{l}\text { Folic acid, vitamin } B_{6} \text { and } \\
\text { vitamin } B_{12}\end{array}$ & UK & $\begin{array}{l}\text { Double-blind, } \\
\text { placebo- } \\
\text { controlled RCT }\end{array}$ & $\begin{array}{l}\text { Inclusion criteria: MCI determined } \\
\text { by TICS-m } \geq 17 \text { and } \leq 29 \text { out of } \\
39 \text {, category fluency test, } \\
\text { MMSE } \geq 24 \text {, CAMDEX, ADL }\end{array}$ & $\begin{array}{l}n 266(\mathrm{~F}=163 \text { and } \\
\mathrm{M}=60) \\
\text { Age }=76 \cdot 7 \text { (sD } 4 \cdot 8) \text { years } \\
\text { (placebo), } 76 \cdot 8 \text { (sD } \\
5 \cdot 1) \text { years (B-vitamin } \\
\text { group) }\end{array}$ & $\begin{array}{l}\text { Duration }=2 \text { years } \\
\text { Placebo or } 0.8 \mathrm{mg} \text { folic } \\
\text { acid, } 20 \mathrm{mg} \text { vitamin } \mathrm{B}_{6} \\
\text { and } 0.5 \mathrm{mg} \text { vitamin } \mathrm{B}_{12} \\
\text { (daily) }\end{array}$ & $\begin{array}{l}\text { Secondary }=\text { MMSE, } \\
\text { HVLT-DR, sematic } \\
\text { memory } \\
\text { (CERAD), CDR, } \\
\text { CLOX, IQCODE }\end{array}$ & $\begin{array}{l}\text { Participants in the treatment group in } \\
\text { the highest tertile of baseline } n-3 \\
\text { fatty acid concentrations benefited } \\
\text { from B-vitamins treatment } \\
\text { compared with placebo for HVLT- } \\
\text { DR }(P=0.047) \text {, CDR score }>0 \text { (\%) } \\
(P=0.043) \text { and CDR (sum of } \\
\text { boxes) }(P=0.04) \text {. In the treatment } \\
\text { group, the highest tertile of } \\
\text { baseline } n-3 \text { fatty acids benefits } \\
\text { from B-vitamin treatment compared } \\
\text { with the lowest tertile for HVLT-DR } \\
(P=0.01) \text { and TICS-m }(P=0.035)\end{array}$ \\
\hline $\begin{array}{l}\text { Van der Zwaluw } \\
\text { et al. }{ }^{(49)}\end{array}$ & Folic acid and vitamin $B_{12}$ & The Netherlands & $\begin{array}{l}\text { Double-blind, } \\
\text { placebo- } \\
\text { controlled RCT }\end{array}$ & $\begin{array}{l}\text { Inclusion criteria: elevated Hcy } \\
(12-50 \mu \mathrm{mol} / \mathrm{l}) \text {, compliant tablet } \\
\text { intake }(\geq 85 \%) \\
\text { Exclusion criteria: cancer } \\
\text { diagnosis in last } 5 \text { years, } \\
\text { bedridden, serum } \\
\text { creatinine }>150 \mu \mathrm{mol} / / \text {, current } \\
\text { or recent use of vitamin } \mathrm{B}_{12} \text { or } \\
\text { folic acid }(>300 \mu \mathrm{mol}) \\
\text { supplements }\end{array}$ & $\begin{array}{l}n \text { 2919, cognitive } \\
\text { subsample: } n 856 \\
\text { ( } F=357 \text { and } M=499) \\
\text { Age }=72 \cdot 6 \text { (sD } 5 \cdot 8 \text { ) years } \\
\text { (placebo), } 72 \cdot 6 \text { (sD } \\
5 \cdot 7) \text { years (B-vitamin } \\
\text { group) }\end{array}$ & $\begin{array}{l}\text { Duration }=2 \text { years } \\
\text { Placebo or treatment } \\
\text { consisting of } 400 \mu \mathrm{g} \text { folic } \\
\text { acid and } 500 \mu \mathrm{g} \text { vitamin } \\
\mathrm{B}_{12} \text {. Both treatments } \\
\text { also contained } 15 \mu \mathrm{g} \text { of } \\
\text { vitamin } \mathrm{D}_{3}\end{array}$ & $\begin{array}{l}\text { Secondary }=\text { MMSE, } \\
\text { RAVLT } \\
\text { Subset: Digit span } \\
\text { forward, Trail } \\
\text { making test I+II } \\
\text { and Letter fluency. }\end{array}$ & $\begin{array}{l}\text { A small improvement was seen in the } \\
\text { treatment group in the MMSE } \\
(P=0.05) \text { but no other statistically } \\
\text { significant advantage was } \\
\text { observed compared with the } \\
\text { placebo group }\end{array}$ \\
\hline Walker et al. ${ }^{(50)}$ & Folic acid and vitamin $B_{12}$ & Australia & $\begin{array}{l}2 \times 2 \times 2 \text { factorial, } \\
\text { double-blind, } \\
\text { placebo- } \\
\text { controlled RCT }\end{array}$ & $\begin{array}{l}\text { Inclusion criteria: elevated } \\
\text { psychological distress } \\
\text { (K10 } \geq 16,<30 \text { ), did not engage } \\
\text { in physical activity (IPAQ) } \\
\text { Exclusion criteria: B-vitamin } \\
\text { supplementation, history of } \\
\text { dementia or bipolar disorder or } \\
\text { suicide risk, } \\
\text { erythrocyte }<250 \mathrm{nmol} / / \text {, vitamin } \\
\mathrm{B}_{12}<130 \mathrm{nmol} / \mathrm{L}, \mathrm{TSH} \\
(0.35-5.0 \mathrm{mU} / \mathrm{l})\end{array}$ & $\begin{array}{l}n 900 \\
\text { Age }=65.97(\mathrm{sD} 4 \cdot 18) \\
\text { years (placebo), } 65.92 \\
(\mathrm{SD} 4.3 \text { ) years (folic } \\
\text { acid }+\mathrm{B}_{12} \text { group) }\end{array}$ & $\begin{array}{l}\text { Duration }=2 \text { years } \\
\text { Placebo or } 400 \mu \mathrm{g} \text { folic acid } \\
\text { and } 100 \mu \mathrm{g} \text { vitamin } B_{12}\end{array}$ & $\begin{array}{l}\text { Primary }=\text { TICS-m, } \\
\text { BTACT survey } \\
\text { and IQCODE }\end{array}$ & $\begin{array}{l}\text { Treatment with B-vitamins led to } \\
\text { improvements in overall cognitive } \\
\text { function score (TICS-m), } \\
\text { immediate }(P=0.046) \text { and delayed } \\
\text { recall }(P=0.013) \text { when compared } \\
\text { with placebo }\end{array}$ \\
\hline
\end{tabular}

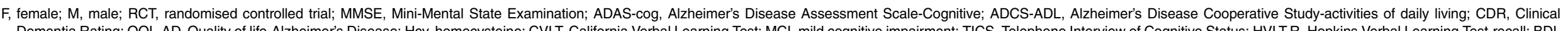

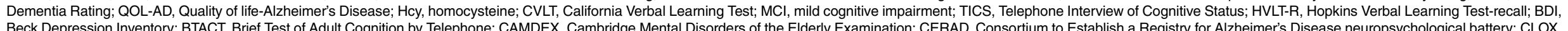

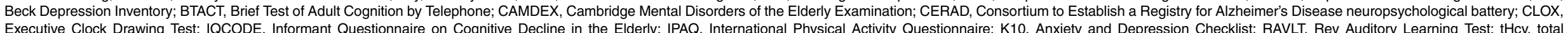
homocysteine; TSH, thyroid-stimulating hormone; WAIS, Wechsler Adult Intelligence Scale. 
Table 3. The effect of $n$-3 PUFA supplementation on cognition in elderly participants (Mean values and standard deviations)

\begin{tabular}{|c|c|c|c|c|c|c|c|c|}
\hline First author & Nutraceutical & Country & Design & Relevant selection criteria & Subjects & Intervention & Cognitive outcomes & Results \\
\hline $\begin{array}{l}\text { Dangour } \\
\text { et al. }{ }^{(51)}\end{array}$ & $\begin{array}{l}\text { DHA and } \\
\text { EPA }\end{array}$ & UK & $\begin{array}{l}\text { Double-blind, } \\
\text { placebo- } \\
\text { controlled } \\
\text { RCT }\end{array}$ & $\begin{array}{l}\text { Exclusion criteria: } \\
\text { diagnoses of diabetes or } \\
\text { dementia, current daily } \\
\text { use of fish-oil } \\
\text { supplements }\end{array}$ & $\begin{array}{l}n 867(\mathrm{~F}=390 \text { and } \mathrm{M}=477) \\
\text { Age }=74.6(\mathrm{sD} 2 \cdot 7) \text { years } \\
\quad(\text { placebo), } 74.7(\mathrm{SD} 2 \cdot 5) \text { years } \\
(n-3 \text { group })\end{array}$ & $\begin{array}{l}\text { Duration }=2 \text { years } \\
\text { Placebo (olive oil) or } \\
\text { treatment consisting of } \\
200 \mathrm{mg} \text { EPA and } \\
500 \mathrm{mg} \text { DHA (daily) }\end{array}$ & $\begin{array}{l}\text { Primary }=\text { CVLT } \\
\text { Secondary = WAIS (short story recall, } \\
\text { processing speed tests, spatial } \\
\text { memory test, digit span backwards, } \\
\text { verbal fluency, prospective memory } \\
\text { tests and digit span forward) }\end{array}$ & $\begin{array}{l}\text { There was no change in cognitive } \\
\text { function scores over } 24 \text { months } \\
\text { and cognitive function did not } \\
\text { decline (all } P>0.05 \text { ) }\end{array}$ \\
\hline $\begin{array}{l}\text { Geleijnese \& } \\
\text { Kromhout }^{(30)}\end{array}$ & $\begin{array}{l}\text { ALA, DHA } \\
\text { and EPA }\end{array}$ & The Netherlands & $\begin{array}{l}2 \times 2 \text { factorial, } \\
\text { double-blind, } \\
\text { placebo- } \\
\text { controlled } \\
\text { RCT }\end{array}$ & $\begin{array}{l}\text { Inclusion criteria: } \\
\text { individuals with stable } \\
\text { MI, MMSE }>21 \\
\text { Exclusion criteria: } \\
\text { institutionalised, } \\
\text { habitual margarine } \\
\text { intake }<10 \mathrm{~g} / \mathrm{d} \text {, habitual } \\
\text { alcohol intake }>6 \\
\text { drinks } / \mathrm{d} \text {, use of fish oils }\end{array}$ & $\begin{array}{l}n 2911 \\
\text { Age }=68 \cdot 9 \text { (sD 5.4) years } \\
\quad \text { (placebo), 69.2 (sD 5.4) years } \\
\text { (EPA-DHA group), 69.1 (sD 5.6) } \\
\text { years (ALA group), 69.2 (sD 5.6) } \\
\text { years (EPA-DHA + ALA group) }\end{array}$ & $\begin{array}{l}\text { Duration: } 40 \mathrm{~m} \\
\text { Placebo or } 20 \mathrm{~g} \text { of } \\
\text { margarine containing } \\
\text { either: (1) } 400 \mathrm{mg} \text { of } \\
\text { EPA-DHA (3:2 ratio), } \\
2.2 \mathrm{~g} \text { of ALA or (3) both } \\
1 \text { and } 2\end{array}$ & Primary outcome $=$ MMSE & $\begin{array}{l}\text { No significant beneficial effects on } \\
\text { cognition in any of the treatment } \\
\text { groups compared with placebo } \\
\text { (all } P>0.05 \text { ) }\end{array}$ \\
\hline Quinn et al. ${ }^{(52)}$ & DHA & USA & $\begin{array}{l}\text { Double-blind, } \\
\text { placebo- } \\
\text { controlled } \\
\text { RCT }\end{array}$ & $\begin{array}{l}\text { Inclusion criteria: Probably } \\
\text { AD (MMSE 14-26), } \\
\text { medically stable, DHA } \\
\text { consumption }<200 \mathrm{mg} / \mathrm{d} \text {, } \\
\text { not taking } n-3 \\
\text { supplements } \\
\text { Exclusion criteria: } \\
\text { medication with } \\
\text { anticholinergic effects or } \\
\text { sedatives or any other } \\
\text { investigational AD } \\
\text { medication }\end{array}$ & $\begin{array}{l}n 402(F=210 \text { and } M=192) \\
\text { Age }=76(\text { sD } 7 \cdot 80 \text { years (placebo), } \\
76 \text { (sD 9.3) years (DHA group) }\end{array}$ & $\begin{array}{l}\text { Duration }=18 \text { months } \\
\text { Placebo or algal-derived } \\
\text { DHA dosed at } 1 \mathrm{~g} \text { twice } \\
\text { daily }\end{array}$ & $\begin{array}{l}\text { Primary = ADAS-cog, CDR sum of } \\
\text { boxes } \\
\text { Secondary = MMSE, ADCS-ADL and } \\
\text { QOL-AD scale }\end{array}$ & $\begin{array}{l}\text { No significant beneficial effects on } \\
\text { cognition in the DHA group } \\
\text { compared with placebo (all } \\
P>0.05 \text { ) }\end{array}$ \\
\hline
\end{tabular}

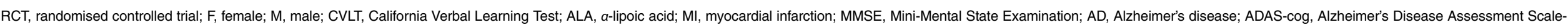
Cognitive; ADCS-ADL, Alzheimer's Disease Cooperative Study-activities of daily living; CDR, Clinical Dementia Rating; QOL-AD, quality of life-Alzheimer's disease; WAIS, Wechsler Adult Intelligence Scale. 
Table 4. The effect of 'Other' nutraceuticals and dietary supplements on cognition in elderly participants (Mean values and standard deviations)

\begin{tabular}{|c|c|c|c|c|c|c|c|c|}
\hline First author & Nutraceutical & Country & Design & Relevant selection criteria & Subjects & Intervention & Cognitive outcomes & Results \\
\hline $\begin{array}{l}\text { DeKosky } \\
\text { et al.(53) and } \\
\text { Snitz et al. }{ }^{(54)}\end{array}$ & $\begin{array}{l}\text { Ginkgo } \\
\text { biloba }\end{array}$ & USA & $\begin{array}{l}\text { Double-blind, } \\
\text { placebo- } \\
\text { controlled } \\
\text { RCT }\end{array}$ & $\begin{array}{l}\text { Exclusion criteria: } C D R>0.5 \text {, several } \\
\text { medications including warfarin medication and } \\
\text { Cholinesterase inhibitors, daily vitamin } \mathrm{E} \\
(>400 \mathrm{IU} ; 10 \mu \mathrm{g}) \text {, history of bleeding disorders } \\
\text { or Parkinson's, abnormal thyroid results, } \\
\text { serum creatinine }>0.02 \mathrm{~g} / \mathrm{l} \text { impaired liver } \\
\text { function }\end{array}$ & $\begin{array}{l}n 3069(\mathrm{~F}=1418 \text { and } \\
\mathrm{M}=1651) \\
\text { Age }=79 \cdot 1 \text { (SD 3.3) years }\end{array}$ & $\begin{array}{l}\text { Duration }=6.1 \text { year } \\
\text { Placebo or twice daily } \\
120 \mathrm{mg} \text { Ginkgo } \\
\text { biloba }\end{array}$ & $\begin{array}{l}\text { Primary = (DeKosky et al.) Incident dementia } \\
\text { and AD } \\
\text { Secondary = Snitz et al.) Composite } z \text { score } \\
\text { including 3MSE, ADAS-cog, TICS, } \\
\text { Cognitive battery (CLVT, Rey Osterrieth } \\
\text { Figure Test, WAIS (revised), Boston } \\
\text { naming test, verbal fluency, trail making } \\
\text { test A and B, and Stroop Colour/Word } \\
\text { Test }\end{array}$ & $\begin{array}{l}\text { No difference in overall incidence rate of } \\
\text { dementia or AD (DeKosky et al.). No } \\
\text { prevention of cognitive decline between } \\
\text { groups (Snitz et al.) }\end{array}$ \\
\hline Dodge et al. ${ }^{(55)}$ & $\begin{array}{l}\text { Ginkgo } \\
\text { biloba }\end{array}$ & USA & $\begin{array}{l}\text { Double-blind, } \\
\text { placebo- } \\
\text { controlled } \\
\text { RCT }\end{array}$ & $\begin{array}{l}\text { Inclusion criteria: no subjective memory } \\
\text { complaint, required score on logical memory } \\
\text { subscale of WAIS, MMSE }>23, \mathrm{CDR}=0 \text {, } \\
\text { free from depressive symptoms } \\
\text { Exclusion criteria: all diseases of the CNS, } \\
\text { vitamin } \mathrm{B}_{12} \text { deficient, thyroid disease, } \\
\text { diabetes, CVD, vision impairment, several } \\
\text { medications }\end{array}$ & $\begin{array}{l}n 118(\mathrm{~F}=72 \text { and } \\
\mathrm{M}=46) \\
\text { Age }=87 \cdot 43 \text { (sD } 2 \cdot 22) \\
\text { years (placebo) } \\
87 \cdot 50 \pm 2 \cdot 14 \text { (Ginkgo } \\
\text { biloba) }\end{array}$ & $\begin{array}{l}\text { Duration }=42 \text { months } \\
\text { Placebo or three time } \\
\text { daily } 80 \mathrm{mg} \text { Ginkgo } \\
\text { biloba }\end{array}$ & $\begin{array}{l}\text { Primary }=\text { Incidence of } \mathrm{MCl} \text { defined as } \\
\text { progression from } \mathrm{CDR}=0-0.5 \\
\text { Secondary }=\text { CERAD Word List Delayed } \\
\text { Recall test, MMSE }\end{array}$ & $\begin{array}{l}\text { No difference in progression to } 0.5 \mathrm{CDR} \\
\text { score between groups. Adjustment for } \\
\text { medication adherence level found lowe } \\
\text { risk of progression to } 0.5 \mathrm{CDR} \text { in } \\
\text { treatment group }(P=0.02)\end{array}$ \\
\hline $\begin{array}{l}\text { Grodstein } \\
\quad \text { et al. }{ }^{(56)}\end{array}$ & $\beta$-Carotene & USA & $\begin{array}{l}\text { Double-blind, } \\
\text { placebo- } \\
\text { controlled } \\
\text { RCT }\end{array}$ & $\begin{array}{l}\text { Inclusion criteria: physicians } \\
\text { Exclusion criteria: history of cancer, active liver } \\
\text { or renal disease, peptic ulcer, or gout }\end{array}$ & $\begin{array}{l}n 4051(\mathrm{M}) \\
\text { Age }=71 \cdot 0 \text { years } \\
\quad \text { (placebo), 70.9 years } \\
\quad(\beta \text {-carotene) }\end{array}$ & $\begin{array}{l}\text { Duration }=1 \text { year } \\
\text { Placebo or } 50 \mathrm{mg} \\
\beta \text {-carotene on } \\
\text { alternate days }\end{array}$ & $\begin{array}{c}\text { Primary }=\text { Global composite score (TICS, } \\
\text { EBMT and Verbal memory score) }\end{array}$ & $\begin{array}{l}\text { Global composite score }(P=0.03) \text {, verbal } \\
\text { memory }(P=0.007) \text { and TICS } \\
(P=0.04) \text { scores improved compared } \\
\text { with placebo but only in participants } \\
\text { that had previously participated in } \\
\text { PHS1 (aspirin study) }\end{array}$ \\
\hline Hu et al. & Melatonin & The Netherlands & $\begin{array}{l}\text { Double-blind, } \\
\text { placebo- } \\
\text { controlled } \\
\text { RCT }\end{array}$ & $\begin{array}{l}\text { Inclusion criteria: residents of homes for elderly } \\
\text { Exclusion criteria: use of monoamine oxidase } \\
\text { inhibitors, long-term NSAID use, severe liver } \\
\text { or kidney dysfunction }\end{array}$ & $\begin{array}{l}n 189(\mathrm{~F}=170 \text { and } \\
\mathrm{M}=19) \\
\text { Age }=85 \cdot 7 \text { (sD } 5 \cdot 6) \text { years }\end{array}$ & $\begin{array}{l}\text { Duration }=3.5 \text { years } \\
\text { Placebo or: }(1) \\
\text { active light } \\
\text { exposure + placebo, } \\
\text { (2) } 2.5 \mathrm{mg} \text { melatonin } \\
\text { and (3) active light } \\
\text { exposure }+2.5 \mathrm{mg} \\
\text { melatonin }\end{array}$ & Primary $=$ MMSE & $\begin{array}{l}\text { In individuals with dementia, melatonin } \\
\text { offered no added benefit to the active } \\
\text { light treatment (all } P>0.05) \text {, however, } \\
\text { change in circadian rhythm (combined } \\
\text { melatonin and light) had a favourable } \\
\text { correlation with MMSE }(P=0.013)\end{array}$ \\
\hline $\begin{array}{l}\text { Riemerma-van } \\
\quad \text { der Lek } \\
\text { et al. }{ }^{(59)}\end{array}$ & Melatonin & The Netherlands & $\begin{array}{l}2 \times 2 \text { factorial, } \\
\text { double-blind, } \\
\text { placebo- } \\
\text { controlled } \\
\text { RCT }\end{array}$ & $\begin{array}{l}\text { Inclusion criteria: residents of homes for elderly. } \\
\text { Exclusion criteria: use of monoamine oxidase } \\
\text { inhibitors, long-term NSAID use, severe liver } \\
\text { or kidney dysfunction, aphakta }\end{array}$ & $\begin{array}{l}n 189(\mathrm{~F}=170 \text { and } \\
\mathrm{M}=19) \\
\text { Age }=85 \text { (sD 5) years } \\
\text { (double placebo), } 86 \\
\text { (sD 5) years } \\
\text { (melatonin only), } 85 \\
\text { (sD 6) years (light } \\
\text { only), } 87 \text { (sD 6) years } \\
\text { (light + melatonin) }\end{array}$ & $\begin{array}{l}\text { Duration }=1 \cdot 25-3.5 \\
\text { years } \\
\text { Placebo or: (1) active } \\
\text { light } \\
\text { exposure + placebo, } \\
\text { (2) } 2.5 \mathrm{mg} \text { melatonin } \\
\text { and (3) active light } \\
\text { exposure }+2.5 \mathrm{mg} \\
\text { melatonin }\end{array}$ & Secondary $=$ MMSE & $\begin{array}{l}\text { In individuals with dementia, melatonin } \\
\text { had no effect on depression ratings } \\
(P>0.05) \text { and may have resulted in } \\
\text { withdrawn behaviour and mood. } \\
\text { However, melatonin attenuated } \\
\text { agitation, reduced sleep latency, } \\
\text { increased sleep duration and greater } \\
\text { uninterrupted sleep. The active } \\
\text { light + melatonin group also } \\
\text { experienced improved sleep efficiency, } \\
\text { sleep length and greater uninterrupted } \\
\text { sleep }\end{array}$ \\
\hline Turner et al. ${ }^{(58)}$ & Resveratrol & USA & $\begin{array}{l}\text { Double-blind, } \\
\text { placebo- } \\
\text { controlled } \\
\text { RCT }\end{array}$ & $\begin{array}{l}\text { Inclusion criteria: probable AD, MMSE } \\
\quad(14-26) \text {, modified Hachinski score }<5 \\
\text { Exclusion criteria: use of resveratrol } \\
\text { supplements, non-AD dementia, sensory } \\
\text { impairment, treatment for diabetes mellitus. }\end{array}$ & $\begin{array}{l}n 104(\mathrm{~F}=68 \text { and } \\
\mathrm{M}=36) \\
\text { Age }=73(\mathrm{sD} 8 \cdot 2) \\
\text { (placebo), 69.8 } \\
\text { (sD 7.7) (resveratrol) }\end{array}$ & $\begin{array}{l}\text { Duration }=1 \text { year } \\
\text { Placebo or resveratrol } \\
\text { at } 500 \mathrm{mg} \\
\text { increments every } \\
13 \text { weeks, ending at } \\
1000 \mathrm{mg} \text { twice daily }\end{array}$ & $\begin{array}{l}\text { Secondary = MMSE, ADAS-cog, ADCS- } \\
\text { ADL, CDR Sum box of boxes, and NPI }\end{array}$ & $\begin{array}{l}\text { No significant differences between groups } \\
\text { on CD Sum ox boxes, ADAS-cog, } \\
\text { MMSE, or NPI. The treatment group } \\
\text { experienced less decline in the ADCS } \\
\text { ADL }(P=0.03)\end{array}$ \\
\hline Vellas et al. ${ }^{(60)}$ & $\begin{array}{l}\text { Ginkgo } \\
\text { biloba }\end{array}$ & France & $\begin{array}{l}\text { Parallel-group, } \\
\text { double-blind, } \\
\text { placebo- } \\
\text { controlled } \\
\text { RCT }\end{array}$ & $\begin{array}{l}\text { Inclusion criteria: previous memory complaint, } \\
\text { MMSE }>25, \mathrm{CAS}<6, \mathrm{GDS}<15 \\
\text { Exclusion criteria: impaired major objective } \\
\text { memory, CDR }>0.5 \text {, dementia diagnosis or } \\
\text { generalised anxiety }\end{array}$ & $\begin{array}{l}n 2854 \\
\text { Age }=76 \cdot 3 \text { (sD } 4.40 \\
\text { years (placebo), } 76 \cdot 3 \\
\text { (sD 4.4) years } \\
\text { (Ginkgo) }\end{array}$ & $\begin{array}{l}\text { Duration }=5 \text { years } \\
\text { Twice per d dose of } \\
120 \mathrm{mg} \\
\text { standardised } \\
\text { Ginkgo biloba } \\
\text { extract (EGb761) or } \\
\text { matched placebo }\end{array}$ & $\begin{array}{l}\text { Primary = Incidence of probable AD } \\
\text { Secondary = Probably AD or mixed } \\
\text { dementia, MMSE, CDR, FCSRT, trail } \\
\text { marking test, verbal fluency, visual } \\
\text { analogue scales and Instruments of ADL }\end{array}$ & $\begin{array}{l}\text { No difference }(P=0.306) \text { in } A D \text { diagnosis } \\
\text { in } A D \text { group }(61) \text { and placebo group } \\
\text { (73) }\end{array}$ \\
\hline
\end{tabular}

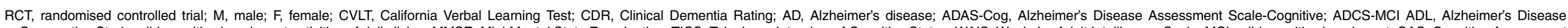

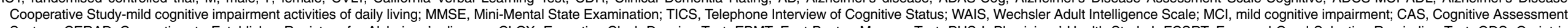

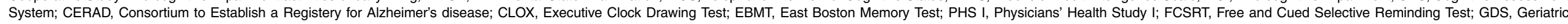
Depression Scale; NPI, Neuropsychiatric Inventory; NSAID, nonsteroidal anti-inflammatory drug; NYU, New York University; RAVLT, Rey Auditory Verbal Learning Test. 
Therefore it is difficult to draw broad conclusions from this study. The initial cognitive testing was conducted face to face; however, the end point tests were performed over the phone, potentially limiting the utility of these findings.

In 2015, a study by Chew et al. ${ }^{(40)}$ assessed cognition in participants with both intermediate and late age-related macular degeneration, consuming either placebo or supplement containing $n-3$ fatty acids DHA and EPA, along with antioxidants lutein and zeaxanthin. Over 5 years (median), this supplementation demonstrated no beneficial cognitive effects as measured by yearly visits and 30-min telephone interview cognitive testing sessions $(P=0.63)$ at 6 months between visits. A strength of this study was the administration of the hearing inventory conducted at the commencement of each bi-annual phone call and the consistent progression of the cognitive tests. Participants were well-nourished and highly educated, but adherence was $75 \%$ during the 5 -year follow-up. A major limitation of this study was that all participants had a neurodegenerative condition, therefore, no significant cognitive improvements was a rather predictive outcome.

A 1-year, 2014 Iranian randomised controlled trial (RCT), supplemented vitamin $\mathrm{C}$ and $\mathrm{E}$ or placebo in elderly individuals with MCI to investigate the effect on cognition as measured by the $\mathrm{MMSE}^{(11)}$. Despite improved biomarkers of oxidative stress and improved performance in the MMSE, no cognitive enhancement was observed in the vitamin group compared with the placebo group $(P=0 \cdot 88)$. The MMSE was administered at three time points during the study, and strength was the homogeneity of the sample to attempt to control for confounding variables. However, despite being conducted in person, the MMSE as the sole cognitive measure is a weakness of this study and perhaps using additional cognitive tests would have uncovered specific cognitive benefits to parallel the improvements in biomarkers. The 1-year length of the study was also a limitation, especially evident by the improvements in MMSE performance by both groups.

Post hoc analysis of 2034 females in the Women's Health Initiative 7-year Ca and vitamin D trial in the USA, revealed no associated between supplementation of calcium carbonate and vitamin $\mathrm{D}_{3}$ with incident dementia or $\mathrm{MCI}^{(43)}$. The primary outcome was the incidence of dementia or MCI, whereas secondary outcomes were measured by MMSE and a cognitive battery (Table 1). No significant differences were observed between groups for dementia risk $(P=0 \cdot 64)$ or MCI $(P=0 \cdot 72)$. A strength of the study was the annual assessment of domainspecific cognitive functions undertaken by a subset of 1420 participants. However, only thirty-nine in the treatment group and thirty-seven in the placebo group developed dementia during the study which limited the statistical power of the analysis and the $10 \mu \mathrm{g} / \mathrm{d}$ dose of vitamin D may not have been adequate to raise serum levels significantly ${ }^{(63)}$. In addition, participants in the placebo group were free to supplement with $\mathrm{Ca}$ and vitamin $\mathrm{D}$ on their terms, and the treatment group consisted of significantly more current or former smokers $(P=0.007)$. These results highlight the relatively unsuccessful outcomes of combined long-term vitamin and antioxidant supplementation to improve cognition. Although the reviewed studies admirably included trials of 5, 7 and 12 years in length, the results do not support combinations of nutraceuticals at the doses investigated.

$B$-vitamins. The hypothesis of an increased homocysteine (Hcy) and B-vitamin status being a risk factor for dementia and $\mathrm{AD}$ has generated nine studies that fell within the inclusion criteria $^{(36,37,44-50)}$, which have been included in this review. A variety of doses and different forms of B-vitamins were used in these studies, and are described in Table 2. All of these studies included vitamin $\mathrm{B}_{12}$ as part of the intervention, with the addition of vitamin $\mathrm{B}_{9}$ (folic acid) included in eight studies ${ }^{(36,37,44,46-50)}$ and vitamin $\mathrm{B}_{6}$ in five studies $(36,37,44,46,48)$. Three included studies ${ }^{(47,49,50)}$ tested the effect of folic acid and vitamin $B_{12}$, whereas one study investigated the effect of vitamin $B_{12}$ alone ${ }^{(45)}$. Between 2006 and 2012, four included studies were published with folic acid, vitamin $\mathrm{B}_{6}$ and vitamin $\mathrm{B}_{12}$ interventions ${ }^{(37,44,46,48)}$. Three of the studies reported increased plasma folate $e^{(44,46,48)}$, two serum folate ${ }^{(36,47)}$ and two erythrocyte folate ${ }^{(37,50)}$. Increased vitamin $\mathrm{B}_{12}$ was reported in eight of the included studies ${ }^{(36,37,44-48,50)}$. Despite reductions in plasma Hcy in eight of the B-vitamin groups in eight of the studies $^{(36,37,44-49)}$, there were limited reports of cognitive improvements in the treatment groups.

The Hcy and B-vitamins in cognitive impairment (VITACOG) study has revealed numerous findings within two studies ${ }^{(36,46)}$ included in this systematic review. The VITACOG trial included relatively high doses of folic acid $(0.8 \mathrm{mg})$, vitamin $\mathrm{B}_{6}(2 \mathrm{mg})$ and vitamin $B_{12}(0.5 \mathrm{mg})$. Hcy was decreased in the treatment group (from 11.3 to $8.7 \mu \mathrm{mol} / \mathrm{l}$ ), and levels of folate, vitamin $\mathrm{B}_{12}$ and holotranscobalamin were all increased. The study by de Jager et al. ${ }^{(46)}$ included individuals with MCI, and reported an improvement in cognition as measured by the Executive Clock Drawing Test compared with placebo $(P=0 \cdot 015)$. This study $^{(46)}$, also found better performance in the MMSE $(P<0.001)$, Hopkins Verbal Learning Test-delayed recall $(P=0.001)$ and semantic memory (category fluency) $(P=0.037)$ tests in those with higher Hcy levels $(>11.3 \mu \mathrm{mol} / \mathrm{l})$. In the same study, an increase of Hcy levels was reported in the placebo group (before: $11.6 \mu \mathrm{mol} / \mathrm{l}$; after: $12.4 \mu \mathrm{mol} / \mathrm{l}$ ), which made the results more reliable and robust. Further analysis from the VITACOG study ${ }^{(36)}$ was published by Oulhaj et al. ${ }^{(36)}$ to analyse plasma concentrations of $n-3$ fatty acids. The groups were categorised into three tertiles, revealing higher performance in the third tertile of the B-vitamin group compared with placebo for episodic memory score $(P=0 \cdot 047)$, better outcome in the CDR score $(P=0.043)$ and CDR sum-of-boxes score $(P=0.04)$. In the $\mathrm{B}$-vitamin group, the highest tertile of baseline $n-3$ fatty acids reported improved performance compared with the lowest tertile in episodic memory score $(P=0 \cdot 01)$ and TICS-m $(P=0 \cdot 035)$. The authors also propose more significant effects with higher baseline concentrations of DHA compared with EPA. However, the authors report this data with the suggestion to use these results for hypothesis generation of future trials due to co-variation between DHA and EPA. Importantly, this study provides data recognising a lack of effect of B-vitamins relative to low baseline plasma 
n-3 fatty acids. Both included VITACOG studies ${ }^{(36,46)}$ were part of a secondary analysis of cognitive function conducted as a follow-up to previous findings of a reduced rate of brain atrophy $(0.76 \%$ /year) in the B-vitamin group compared with placebo $(1.08 \% / \text { year })^{(64)}$. Despite these significant findings, these are still only preliminary results, and larger studies are required.

Out of all eight included studies, the two largest studies by van der Zwaluw et al. ${ }^{(49)}$ ( $n$ 2919; cognitive subsample: $n$ 856) and Walker et al. ${ }^{(50)}$ ( $n$ 900) both reported small improvements in cognition. The study by van der Zwaluw et al. ${ }^{(49)}$ reported a significant improvement in the MMSE $(P=0.05)$ by the treatment group compared with the placebo group (MMSE decline of only 0.1 in the treatment group and 0.3 in the placebo group). Walker et al. ${ }^{(50)}$ detected an improvement in overall cognitive function score in a general population sample with elevated psychological distress. Participants were assessed by the TICS-m $(P=0.032)$, with the treatment group reporting improvements in immediate $(P=0.046)$ and delayed recall $(P=0.013)$ when compared with the placebo group at 24 months ${ }^{(50)}$. It should be noted that TICS is not as sensitive as an in-person interview. The study by Walker et $a l .{ }^{(50)}$ reported increased Hcy over 24 months, although this increase was lower $(P<0.001)$ in the treatment group (from 9.6 to $10.4 \mu \mathrm{mol} / \mathrm{l}$ ), compared with the placebo group (from $9 \cdot 8$ to $12 \cdot 0 \mu \mathrm{mol} / \mathrm{l}$ ). Both interventions consisted of $400 \mu \mathrm{g}$ folic acid. However, van der Zwaluw et $a l{ }^{(49)}$, used $500 \mu \mathrm{g}$ of vitamin $\mathrm{B}_{12}$ compared with Walker et $a l .{ }^{(50)}$ who used $100 \mu \mathrm{g}$. These two studies incorporated the lowest dose of folic acid $(400 \mu \mathrm{g})$ used in the eight included RCT, suggesting that smaller doses potentially provide benefit to the elderly. The overall conclusions in the additional five studies did not report cognitive improvements with B-vitamin treatment ${ }^{(37,44,45,47,48)}$. However, Kwok et al. ${ }^{(47)}$, reported reduced rate of cognitive decline in the Mattis dementia rating scale with B-vitamins in individuals with plasma Hcy $>13 \mu \mathrm{mol} / 1$ $(P=0.003)$.

There were several limitations apparent in these studies. Four included studies included participants with mean Hcy levels $>13 \mu \mathrm{mol} / 1$ at baseline ${ }^{(37,45,48,49)}$. Only two of these ${ }^{(45,48)}$ including individuals with mean levels considered to be hyperhomocysteinaemia at baseline $(>15 \mu \mathrm{mol} / \mathrm{l})$. Five of the included studies did not include participants that had experienced a cognitive decline ${ }^{(37,45,48-50)}$. The two VITACOG studies included participants with $\mathrm{MCI}^{(36,46)}$, and only two studies included participants with dementia ${ }^{(44,47)}$. The placebo groups in the studies did not experience cognitive decline, limiting the ability for meaningful statistical comparisons to be made. Furthermore, the use of the MMSE by six of the studies ${ }^{(37,45,46,48-50)}$, is not suitable to detect subtle changes in cognition. Four of the studies excluded individuals based upon low blood baseline B-vitamin levels ${ }^{(44,45,47,50)}$. Several studies referred individuals with low blood B-vitamin levels for medical treatment. It is possible that supplementation with B-vitamins only benefits those with low B-vitamin status. However, only the study ${ }^{(45)}$ of vitamin $B_{12}$ by Dangour et al. ${ }^{(45)}$ used moderately deficient participants. Some heterogeneity of blood B-vitamin levels may have occurred in the studies not excluding participants due to baseline B-vitamin levels ${ }^{(37,46,48,49)}$.
It is also noteworthy that mandatory folic acid fortification has been enacted in the USA since 1998. The inclusion of synthetic folic acid in foods may have contributed to the overall intake of participants in the study by Aisen et $a l^{(44)}$, thus lessening any potential effect on cognition ${ }^{(44)}$. Aisen et al. ${ }^{(44)}$ also included individuals consuming B-vitamins (folic acid in multivitamin $<400 \mu \mathrm{g} / \mathrm{d}$ ), as did the VITACOG studies ${ }^{(36,46)}$ (folic acid $<300 \mu \mathrm{g} / \mathrm{d}$, pyridoxine $<3 \mathrm{mg} / \mathrm{d}$ and vitamin $\mathrm{B}_{12}<3 \mathrm{mg} / \mathrm{d}$ ) and Van Der Zwaluw et al. ${ }^{(49)}$ (folic acid $<300 \mu \mathrm{mol} / \mathrm{l}$ ). In one study ${ }^{(47)}$, it was unclear whether individuals consuming B-vitamins were excluded. Larger sample sizes may be required to assess the potential of B-vitamin formulations to improve cognition in the elderly. It has also been suggested that focus on only vitamins $\mathrm{B}_{6}, \mathrm{~B}_{9}$ and $\mathrm{B}_{12}$ has ignored the impact of the lesser known B-vitamins involved in Hcy metabolism that may preserve brain health ${ }^{(65)}$. In summary, while the link between B-vitamin intake and blood levels of Hcy and folate with dementia is well recognised, the benefits due to B-vitamin supplementation remain unclear. However, research involving the interactions between the B-vitamins and biomarkers, relative to other nutrients, nutraceuticals such as the $n-3$ fatty acids ${ }^{(36)}$, and genetic polymorphisms ${ }^{(66)}$ remain an important area of focus.

n-3 PUFA. A study by Dangour et al. ${ }^{(51)}$ conducted a 2-year trial of daily $200 \mathrm{mg}$ EPA and $500 \mathrm{mg}$ DHA supplementation in comparison to an olive oil placebo in cognitively healthy adults. Participant cognition did not decline during this time (Table 3), and no benefit was seen in the treatment group $(P>0.05)$. The strengths of this study ${ }^{(51)}$ included measurement of significantly higher serum EPA (treatment: 49.9 (SD 2.7) mg/l; placebo: 39.1 (sD 3.1) $\mathrm{mg} / \mathrm{l} ; P=0.009$ ) and DHA (treatment: 95.6 .9 (sD 3.1) $\mathrm{mg} /$ 1; placebo: 70.7 (SD 2.9) $\mathrm{mg} / 1 ; P<0.001$ ) concentrations in the treatment group, but this suggests that higher serum levels are not associated with improved cognition. The choice of olive oil as a placebo is questionable, as olive oil itself is known to contain bioactive compounds and have beneficial health properties ${ }^{(67)}$ and as such has been associated with improved cognition $^{(68)}$. As participants were cognitively healthy and there was a lack of decline in the control arm, it is likely that 2 years was not sufficient for any cognitive benefits to be observable.

Likewise, Geleijnse et al. $^{(30)}$ found no benefit of daily supplementation of $400 \mathrm{mg}$ EPA and DHA (3:2 ratio) added to $20 \mathrm{~g}$ of margarine compared with placebo in stable individuals having previously suffered a myocardial infarction. In addition, $2 \mathrm{~g}$ of $\alpha$-lipoic acid (ALA) did not result in cognitive benefits $(P=0.44)$, nor did a combination of ALA and the $n-3$ fatty acids in the margarine $(P=0 \cdot 12)$. Certainly, strengths of this study were its large sample size ( $n$ 2911), a 40-month study duration and especially reporting that no patients were lost to follow-up. In addition, participants were instructed to not use the margarine in cooking or baking, limiting the likelihood that the margarine would be degraded under high heat conditions and then consumed. However, the main limitation of this study was the sole use of MMSE as the measure of cognitive outcome. The study also employed a moderate dose of EPA and DHA, although shorter-term studies with higher doses have yielded mixed results ${ }^{(69,70)}$. Furthermore, the margarine also contained 
other fatty acids including SFA palmitic acid and stearic acid as well as trans-fatty acids, which could lessen any potential benefits. The margarine was consumed with 4.3 slices of bread per $\mathrm{d}$, potentially confounding the results further, although the authors state that this level of bread consumption is common in the study recruitment area.

The final study, Quinn et al. ${ }^{(52)}$ supplemented with a higher dose than the previous two studies, consisting of $1 \mathrm{~g}$ of algalderived DHA twice daily (2 $\mathrm{g}$ total) for 18 months. Again, no significant benefits were observed in the treatment group compared with placebo, with improvements seen in the ADAS$\operatorname{cog}$ in both groups. As measured in the VITACOG study ${ }^{(64)}$, the rate of brain atrophy was also measured by volumetric MRI in a subsample of 102 participants with no reduction in the rate of total volume brain decline being observed compared with placebo. A strength of this study ${ }^{(52)}$ was the high dose use of DHA, however, as a plant source of DHA, it may not be absorbed as well as fish sources of $\mathrm{DHA}^{(71)}$. Despite this, plasma DHA (and a subsample of cerebrospinal fluid (CSF)) was increased in the treatment group. Interestingly, when APOE4 carriers were excluded from the analysis ( $n$ 232), APOE4 negative participants $(n 170)$ had a slower rate of decline in the ADAS-cog and MMSE compared with the placebo group (both, $P=0.03$ ) but was not evident in other measures. As this study consisted of a high percentage of $A P O E 4$ carriers $(57 \cdot 7 \%)$, this analysis sets the stage for future trials to conduct such subgroup analysis. A limitation of this study was the high attrition rate of $28 \%$ in the DHA group and $24 \%$ of the placebo group not completing the study with the main reason reported being due to adverse effects. The included studies have found only minor benefits to $n-3$ fatty acid supplementation yet future long-term trials with higher doses, and consideration of the APOE4 genotype may promote cognitive benefits.

Other nutraceuticals ( $\beta$-carotene, melatonin, resveratrol and Ginkgo biloba). As part of the Physicians' Health Study II, 4051 physicians consumed placebo or $50 \mathrm{mg}$ of $\beta$-carotene on alternate days and undertook cognitive testing by telephone ${ }^{(56)}$. Analysis following 1 year of supplementation found improved performance in global and verbal memory but only in participants who also took part in the Physicians' Healthy Study I (all $\left.P^{\prime} \mathrm{S}<0.05\right)$ which found positive effects from aspirin use ${ }^{(61)}$. Strengths of this study ${ }^{(56)}$ included a dose of $\beta$-carotene being equivalent to that found in over ten medium sized carrots, and high participation rates (over $92 \%$ in both groups). The relatively short 1-year follow-up was a limitation of this study as cognitive testing only commenced shortly before the $\beta$-carotene arm was terminated and not all baseline cognitive data were available. In addition, various confounders apply to the use of physicians as participants in cognitive studies as previously discussed.

Two included studies investigated the long-term use of melatonin and active light therapy in the same, mostly female, Netherlands nursing home dementia participants ${ }^{(57,59)}$. Although melatonin is considered to be a pharmacologic agent in many jurisdictions, it is also commonly sold as an over the counter supplement to assist with sleep quality, and in Canada it is regulated as a natural health product ${ }^{(72)}$. One study ${ }^{(59)}$ reported that bedtime melatonin only treatment had no effect on cognition as measured by the MMSE $(P>0.05)$. However, some benefits were observed regarding sleep duration and fewer sleep disruptions when combined with light therapy. Another study ${ }^{(57)}$ reported no benefit of bedtime melatonin supplementation, only chronic effects of treatment as it applied to changes of temporal correlations with physical activity. The lack of cognitive improvements observed in these studies may be due to the sole use of the MMSE as a cognitive measure but is likely attributable to most participants being in the late stages of dementia (age: 85.7 (SD 5.6) years). This trial was a novel long-term investigation of active light therapy on the circadian rhythm, sleep and activity in dementia patients. The authors noted that the dose of melatonin $(2.5 \mathrm{~g})$ may be too high and thereby impacted on mood behaviour.

The study by Turner et al. ${ }^{(58)}$ investigated whether an escalating dose of resveratrol in individuals with $\mathrm{AD}$ affected levels of A $\beta 40$ and brain volume. Participants in the treatment group commenced the trial with a $500 \mathrm{mg}$ dose. The dose was increased every 13 weeks resulting in a final dose of $1000 \mathrm{mg}$ twice daily during weeks 39 and 52. Resveratrol may cause gastrointestinal stress at higher doses, but in this study it was well tolerated by participants with no significant differences between groups for adverse events aside from weight loss $(P=0.038)$ in the treatment group $(-0.92(\mathrm{sD} 4.9) \mathrm{kg})$ compared with the placebo group $(+0.54(\mathrm{sD} 3.2) \mathrm{kg})$. The study was underpowered to detect differences in the secondary cognitive outcomes that were measured which included MMSE and the ADAS-cog. However, no benefits were identified in the treatment group except less decline in the activities of daily living score $(P=0.03)$. Greater reductions in levels of $\mathrm{CSF}$ A $\beta 40$ and plasma $\mathrm{A} \beta 40$ were identified in the placebo group $v$. the treatment group ( $P=0.002$ and $P=0.024$, respectively). The level of $\mathrm{A} \beta 40$ has been shown to decrease as dementia advances; hence there may be a role for resveratrol supplementation to ameliorate this decline. The results also provide evidence that resveratrol crosses the blood-brain barrier as resveratrol metabolites were measurable in the CSF. However, neuroimaging found that the treatment group had greater brain volume loss $(P=0.025)$, and higher ventricular volume compared with the placebo group $(P=0.05)$. Ventricular enlargement has been proposed to be an important short-term marker of AD severity, including risk of progression from $\mathrm{MCI}$ to $\mathrm{AD}^{(73)}$. A key strength of this study was the inclusion of analysis by $A P O E 4$ genotype which revealed an effect of resveratrol on plasma $\mathrm{A} \beta 40$ in the $A P O E 4$ carriers $(P=0 \cdot 04)$, and a greater decline in brain volume $(P=0 \cdot 02)$. More research is required to understand the significance of the findings, including if the loss in brain volume is related to the potential weight loss properties of resveratrol. A larger, longer study, powered to assess cognitive outcomes will better examine if the loss in brain volume is related to a reduction in inflammation and brain swelling.

Ginkgo biloba has been studied extensively for its potential health benefits and four publications reporting on three trials fulfilled our inclusion criteria. Two publications by DeKosky et $a l .{ }^{(53)}$ and Snitz et al. ${ }^{(54)}$ investigated 3069 individuals using a twice daily dose of $120 \mathrm{mg}$ Ginkgo biloba extract for a medium follow-up of $6 \cdot 1$ years. At enrolment, thorough exclusion criteria 
were applied. The majority of participants ( $n$ 2587) were determined to have normal cognition and 482 were classified as having MCI. Dekosky et al. reported no difference in incidence of dementia between the placebo group ( $n$ 246) and the treatment group ( $n$ 277). Cognitive improvements were measured by composite $z$ score as published by Snitz et al. ${ }^{(54)}$, and also found no benefit to annual rates of cognitive decline. The rates of dropout for the study were $6.3 \%$, which may be considered strength of the study, however, adherence to both assignments dropped to $60 \cdot 3 \%$ of participants by the end of the study. Compliance was similar between groups but this may be a strong source of bias confounding the final results.

The 2012 study by Vellas et al. ${ }^{(60)}$ found no reduction in the risk of progression to $\mathrm{AD}$ over 5 years in participants that reported memory complaints to their physician. The study was a large and age-matched with the treatment group also receiving $120 \mathrm{mg}$ of standardised Ginkgo biloba extract for consumption twice per $d$. The strength of this study includes the involvement of physicians in identifying participants that may be at risk of developing $\mathrm{AD}$. However, progression to $\mathrm{AD}$ was only observed in sixty-one participants in the treatment group, and seventythree in the placebo group, out of 2854 individuals with an average age of 76.3 years (both groups, SD \pm 4.4 ). Another strength is the exploratory subgroup analysis that tested for relationships between progression of $\mathrm{AD}$ and some factors including APOE4 positive status and hypertension at baseline. The analysis found fewer males progressed to AD with treatment (treatment $=14$, placebo $=32, P=0 \cdot 011$ ) The authors suggested the overall absence of protective effect in this study may have been due to the higher level of education in the sample compared with the general population and exclusion of individuals receiving an MMSE score $<25$. In the smallest included Ginkgo biloba study ( $n$ 118), Dodge et al. ${ }^{(55)}$ applied a dose of $80 \mathrm{mg}$ three times daily, and found no difference between groups in progression from a CDR score of 0 to 0.5 over 42 months. Secondary analysis, found benefit when controlling for baseline medication adherence during the first 6 months of the study (hazard ratio $=0.33 ; 95 \%$ CI $0.12,0.89$ ) for progression to 0.5 CDR. This analysis demonstrated there may be benefit to older individual (mean age $>87$ years in both groups), however, concern was expressed for seven cases of stroke/transient ischaemic attack in the treatment group compared with none in the placebo group. All included Ginkgo biloba studies included individuals in late life, therefore, it is plausible that benefits may exist when supplementing earlier in the life. In addition, the trials also did not include individuals with dementia. A 1997 study found modest improvements with $120 \mathrm{mg} / \mathrm{d}$ Ginkgo extract in the ADAS-cog $(P=0.04)$ in individuals with mild to severe $\mathrm{AD}^{(74)}$, therefore it is premature to rule out Ginkgo biloba as an effective treatment to improve cognition.

The remaining articles in this review not categorised in the first three sections found limited overall evidence of efficacy in longterm trials. Despite this, further research is warranted to investigate melatonin in younger at-risk individuals, whereas questions remain regarding the usefulness of Ginkgo biloba and resveratrol to treat or prevent impaired cognition.

At this point, it should be mentioned that no attempt was made to meta-analyse the data, due to the heterogeneity of the included studies and cognitive assessment tools used, as well as the number of different nutraceuticals listed in the systematic review.

\section{Discussion}

The use of nutraceuticals and dietary supplements with a longterm view to improving cognition in elderly individuals aged over 65 years is not supported by the currently published data. This review included randomised, double-blinded, placebocontrolled clinical trials. Only trials consisting of over 100 participants were included in an attempt to make inferences about data from studies that had more statistical power. In addition, the duration of all included studies was between 1 and 12 years, again strengthening the overall findings of this review through the exclusion of short-term studies. Long-term nutritional trials are particularly difficult to conduct due to the associated costs, yet they represent an important aspect of nutrition research due to the time it can take for positive or adverse effects to become symptomatic. There was an overall low risk of bias for the majority of studies included (Table 5). However, the longerterm focus of included studies in this review still failed to find compelling evidence that currently warrants the use of dietary supplements and nutraceuticals in the elderly. Of the included literature, none reported notable memory enhancement in the participants, and evidence of maintenance of memory function was scant at best. In addition, no studies reported a reduction in the progression towards MCI or dementia.

The RCT is generally regarded as the highest quality clinical trial study design in nutrition ${ }^{(75)}$, however, there are several general limitations to be considered when evaluating the ability of nutraceuticals and dietary supplements to improve cognition in the included studies (Table 5). Many included studies investigated cognitively healthy people who may not experience cognitive decline in life but may instead suffer mortality from another cause ${ }^{(76)}$. The included studies examined individuals aged over 65 years during a stage of their life where the health quality may have already started to diminish. Importantly, nutraceutical interventions may be more effective if treatment begins earlier in life. In the case of B-vitamin supplementation, benefits resulting from 3 years of treatment have been observed in older individuals under 65 years that were recruited based on elevated Hcy levels $(>13 \mu \mathrm{mol} / \mathrm{l})^{(77)}$. RCT investigating nutraceutical intake in individuals already diagnosed with dementia and $\mathrm{AD}$ may be undertaken too late to be able to measure noticeable changes in neuropsychological or pathological features. Moving forward, the focus of long-term dietary supplements and nutraceutical use should focus longitudinally on individuals at increased risk of developing MCI or $\mathrm{AD}$ at an earlier life stage. This review has suggested mixed results with benefits to cognition associated with B-vitamin use especially in studies with larger cohorts and benefits related to $n$-3 fatty acids. The VITACOG study has revealed reduced brain atrophy with B-vitamin use ${ }^{(64)}$, and an important recent finding suggesting that efficacy of B-vitamins to improve cognition may be dependent on plasma $n-3$ fatty acid status ${ }^{(36)}$. DHA was also found to have a greater effect on cognition in individuals without a copy of the APOE4 gene ${ }^{(52)}$. In addition, the quality of 
Table 5. Risk of bias summary for studies included in this systematic review (Mean values and standard deviations)

\begin{tabular}{|c|c|c|c|c|c|c|c|}
\hline Bias category & $\begin{array}{l}\text { Random sequence } \\
\text { generation } \\
\text { (selection bias) }\end{array}$ & $\begin{array}{c}\text { Blinding of participants } \\
\text { and personnel } \\
\text { (performance bias) }\end{array}$ & $\begin{array}{l}\text { Blinding of outcome } \\
\text { assessment (detection } \\
\text { bias) (mortality) }\end{array}$ & $\begin{array}{l}\text { Incomplete data } \\
\text { addressed } \\
\text { (attrition bias) }\end{array}$ & $\begin{array}{c}\text { Selective } \\
\text { reporting } \\
\text { (reporting bias) }\end{array}$ & $\begin{array}{l}\text { Other } \\
\text { bias }\end{array}$ & $\begin{array}{l}\text { Overall } \\
\text { Bias }\end{array}$ \\
\hline \multicolumn{8}{|c|}{ Combinations of different nutraceuticals } \\
\hline Chew et al. $(2015)^{(40)}$ & Low & Low & Low & Low & Low & Low & Low \\
\hline Grodstein et al. (2013) & Low & Low & Unclear & Moderate & Low & Low & Low \\
\hline McNeil et al. (2007) & Low & Low & Low & Moderate & Low & Low & Low \\
\hline Naeini et al. $(2014)^{(11)}$ & Unclear & Unclear & Unclear & Unclear & Low & Low & High \\
\hline Rossum et al. (2012) & Low & Low & Unclear & Moderate & Low & Low & Low \\
\hline \multicolumn{8}{|l|}{ B-vitamins } \\
\hline Aisen et al. (2008) $)^{(44)}$ & Low & Low & Low & Moderate & Low & Moderate & Low \\
\hline Dangour et al. (2015) ${ }^{(45)}$ & Low & Low & Low & Low & Low & Low & Low \\
\hline de Jager et al. (2012) $)^{(46)}$ & Low & Low & Low & Low & Low & Low & Low \\
\hline Ford et al. $(2010)^{(37)}$ & Low & Low & Low & Moderate & Low & Low & Low \\
\hline Kwok et al. $(2011)^{(47)}$ & Unclear & Low & Unclear & Moderate & Low & Low & Moderate \\
\hline McMahon et al. (2006) $)^{(48)}$ & Moderate & Unclear & Unclear & Low & Low & Low & Moderate \\
\hline Oulhaj et al. $(2016)^{(36)}$ & Low & Low & Unclear & Low & Low & Low & Low \\
\hline Van Der Zwaluw et al. (2014) $)^{(49)}$ & Low & Low & Low & Moderate & Low & Low & Low \\
\hline Walker et al. $(2012)^{(50)}$ & Low & Low & Low & Low & Low & Low & Low \\
\hline \multicolumn{8}{|l|}{$n-3$ PUFA } \\
\hline Dangour et al. (2010) ${ }^{(51)}$ & Moderate & Low & Low & Low & Low & Low & Low \\
\hline Geleijnse et al. (2012) & Unclear & Unclear & Unclear & Low & Low & Low & Moderate \\
\hline Quinn et al. $(2010)^{(52)}$ & Low & Low & Low & Moderate & Low & Low & Low \\
\hline \multicolumn{8}{|l|}{ Other } \\
\hline $\begin{array}{l}\text { DeKosky et al. }(2008)^{(53)} \text { and } \\
\text { Snitz et al. }(2009)^{(54)}\end{array}$ & Low & Moderate & Moderate & High & Low & Low & High \\
\hline Dodge et al. $(2008)^{(55)}$ & Unclear & Low & Low & Low & Low & Low & Low \\
\hline Grodstein et al. $(2007)^{(56)}$ & Low & Low & Unclear & Low & Low & Low & Low \\
\hline Hu et al. $(2016)^{(57)}$ & Low & Low & Low & Low & Low & Low & Low \\
\hline Turner et al. $(2015)^{(58)}$ & Low & Low & Low & Low & Low & High & Moderate \\
\hline $\begin{array}{l}\text { Riemersma-van der Lek et al. } \\
(2008)^{(59)}\end{array}$ & Low & Low & Low & Low & Low & Low & Low \\
\hline Vellas et al. $(2012)^{(60)}$ & Low & Low & Low & Moderate & Low & Moderate & Low \\
\hline
\end{tabular}

supplemental fish oil is a determinant of their health effects ${ }^{(78)}$. Taken together, these findings highlight the complicated nature of elucidating the potential benefits of individual nutrients and categories of nutrients, relative to blood biomarkers and genetic risk factors. This systematic review has uncovered only limited evidence supporting the use of nutraceuticals and dietary supplements to improve cognition in the elderly. However, future trials sufficiently powered to investigate specific cognitive risk factors are likely to find more benefits in particular groups including the elderly.

When assessing the viability of nutraceutical and dietary supplement use in the elderly, a number of considerations must be taken into account. Firstly, there exists a poorly defined area between the use of specific compounds from food that potentially assert health benefits, and those that are considered formally as pharmaceutical agents. Synthetic versions of nutraceuticals may hold different physiological properties than naturally occurring forms found in food ${ }^{(79)}$. Second, all trials included in this review used sample populations at varying life stages and degrees of cognitive health, as indicated by the heterogeneity between selection criteria. For example, MMSE cut-offs varied throughout studies with $>23$ being considered to exclude significant cognitive impairment in one study ${ }^{(55)}$, and 24 in another ${ }^{(45)}$. In one study ${ }^{(11)}$, participants were classified to have MCI with an MMSE score between 21 and 26, whereas another classified probable $\mathrm{AD}$ as being between 14 and $26^{(52)}$. Variation between different stages of cognitive impairment across the included trials makes it difficult to make conclusions regarding the overall usefulness of the nutraceuticals. Only seven studies in this review included individuals with dementia $^{(44,47,52,57-59)}$, and three included studies ${ }^{(11,36,46)}$ used individuals with MCI. Certainly, there is difficulty in conducting trials in individuals with cognitive impairment and several studies in this review required caregiver assistance or approval to participate from a proxy. It is unrealistic to expect trials conducted worldwide to utilise the same selection criteria, however, standardised criteria would be highly advantageous moving forward to assist in determining the cognitive outcomes in nutritional intervention studies. This may include standardised goal criteria for the baseline cognitive status, a hierarchy of suitable cognitive outcome measures, and also guidelines to inclusion criteria based on important biomarkers. Further, there exists difficulty in assessing the optimal doses for treatment with nutraceuticals and supplements. For example, folate toxicity may be breached through supplementation, a relatively high folate containing diet, and also the addition of folic acid through fortified foods in many countries. Although folic acid fortification has been determined important to prevent neural tube defects, risks of excessive B-vitamin intake exist with risk of chromosomal and nerve damage, particularly with certain gene variants ${ }^{(80)}$. Moreover, individuals with normal serum vitamin $\mathrm{B}_{12}$ and high folate levels have been demonstrated to be more likely to have impaired cognition ${ }^{(81)}$.

Genetics play a significant role in cognitive performance in the elderly. The APOE4 gene is not only a major risk factor for $\mathrm{AD}$ but even in non-demented individuals, it predicts a higher 
rate of decline in verbal memory and abstract reasoning ${ }^{(82)}$. The studies in this review mostly overlooked the effect of the APOE4 genotype on cognition in their analysis. Quinn et al. ${ }^{(52)}$ found that when APOE4 genotype was excluded, a slower rate of cognitive decline was observed in participants that did not carry the APOE4 gene ${ }^{(52)}$. As APOE4 is recognised as the most powerful genetic predictor of $\mathrm{AD}^{(83)}$, it is possible that $A P O E$ genotype is confounding the results in some studies and that greater benefits may have been observed in the treatment groups with APOE4 carriers excluded. Nutrient status in the brains of $A P O E 4$ carriers may differ, as evident by differences in Se concentrations in $\mathrm{AD}$ brains of $A P O E 4$ carriers $^{(84)}$. In addition to $A P O E$ genotype, several different polymorphisms of the fatty acid desaturase (FADS) gene cluster compromise $n-3$ status by slowing the conversion of alpha-linolenic acid to EPA and $\mathrm{DHA}^{(85)}$. Other FADS variants also increase the number of longchain PUFA that may be synthesised from plant-based foods ${ }^{(86)}$. Although expression of FADS may be moderated hormonally ${ }^{(87)}$, further research is required to determine if there is a causal relationship between FADS and $n$-3 fatty acid metabolism. Higher intakes of $n-3$ fatty acids may be required to elicit benefits in individuals with specific FADS polymorphisms. Future studies on the effects of nutraceuticals on cognition in specific genetic subgroups are warranted, particularly in APOE4 carriers, due to the relatively high number of individuals diagnosed with $\mathrm{AD}^{(88)}$.

Presently, older people and their carers must apply caution when considering the use of nutraceuticals and dietary supplements. The majority of nutraceuticals consumed at the recommended doses are generally regarded as safe in most cases ${ }^{(89)}$. However, toxic side effects are still common, and caution is advised $^{(28,90)}$, including their potential to interact with prescribed drugs ${ }^{(23)}$. Relative to cognition, only a limited number of nutraceuticals have been systematically studied longterm for safety. Numerous studies have found no benefit to mortality from dietary supplements and nutraceutical use. In fact, a 2013 review of seventy-eight $\mathrm{RCT}^{(91)}$ suggested that $\beta$-carotene, vitamin $\mathrm{E}$ and high dose vitamin A may be associated with increased all-cause mortality in participants with an average age of 63 years (range: 18-103). Meta-analyses of multivitamin-mineral supplementation and vitamin $\mathrm{E}$ usage (up to $5500 \mathrm{IU} / \mathrm{d}$ ) both reported no effect on all-cause mortality ${ }^{(92,93)}$. Another study ${ }^{(26)}$ found that greater nutraceutical and dietary supplements usage may be associated with an increased total mortality risk, particularly in association with iron supplementation. However, a small cohort study suggested that antioxidant vitamins may reduce cancer and all-cause mortality ${ }^{(94)}$, with a 2013 systematic review ${ }^{(95)}$ also reporting on two trials with a small significant benefit from multivitamins on cancer in men. Overall, there is a lack of high-quality evidence to support the long-term use of nutraceuticals and dietary supplements, especially in those over 65 years of age, and epidemiological evidence suggests that their use may even be detrimental to health ${ }^{(90)}$

It is also noteworthy that a number of important published trials did not fall under our inclusion criteria. The nutraceutical formulation Souvenaid® (Nutricia N.V.) includes ingredients hypothesised to support synaptogenesis such as $n$ - 3 fatty acids,
$\mathrm{Cl}$ and uridine monophosphate, and has been shown to promote improvements in verbal recall in individuals with early $\mathrm{AD}^{(96)}$. A larger and longer trial of Souvenaid® (Nutricia N.V.) is ongoing, and preliminary results have shown promise with reduced brain atrophy reported ${ }^{(97)}$. In addition, the recent 3-year Multidomain Alzheimer Preventative Trial ${ }^{(98)}$ was published outside of our included search dates. Despite being one of the most comprehensive lifestyle and cognition studies conducted to date, only very minimal benefits were found comparing a multidomain intervention (cognitive training, nutritional counselling and physical activity) and $n-3$ fatty acids (225 mg EPA and $800 \mathrm{mg}$ DHA), $n$-3 fatty acids alone, multidomain intervention with placebo, to placebo alone. In response to this study, it has been suggested that higher dose DHA of $2 \mathrm{~g}$ for longer than 3 years is necessary to promote an increase in CSF levels of DHA to promote dementia prevention ${ }^{(99)}$. Finally, a study investigating curcumin including ninetysix participants found mild benefits in the Montreal Cognitive Assessment in a 12-month $\mathrm{RCT}^{(38)}$. This finding is expected to compliment upcoming trials exploring combined effects of curcumin with yoga, and curcumin with fish oil.

\section{Limitations of cognitive tests administered}

The study of cognition and cognitive decline is fraught with challenges. Although several measures of cognitive function are available, these tests rely on significant time spent on administration, which may not be feasible for use in research settings. In such contexts, brief screening measures are favoured. Screening measures allow for the detection of cognitive disorders without the need for significant time spent completing actions and is the preferred method of detecting cognitive decline and dementia within large-scale studies. However, these screening measures do not allow for the detection of subtle and domain-specific cognitive changes. Although some studies made efforts to detect changes in specific cognitive domains through the use of domain-specific measures such as those that included Wechsler Adult Intelligence Scale-IV subscales, many studies relied upon broad screening measures (such as the MMSE) which lack sensitivity in the detection of domain-specific change and cognitive impairment. In the research setting, it is cost and time-prohibitive to conduct a thorough battery of cognitive tests to determine the cognitive state of participants fully. As evident in this review, the most common of these is the MMSE, followed by the TICS and ADASCog. Most of the included studies conducted the tests over the telephone, which is not as effective as face-to-face assessment. Even considering these obvious limitations, brief instruments such as these are recognised to detect dementia ${ }^{(66)}$ adequately, but despite considerable effort in some studies, the majority included in this review may not have been suitably designed to detect subtle, but significant, changes in cognitive performance. It is also possible that detection of slowed rates of overall decline, as well as buffering of decline from nutraceutical use, was not measurable in the majority of the studies. Lastly, the dropout rates varied across the included studies and a small number considered the cause of death (by dementia) in the analysis. However, overall, it can be speculated that some 
participants did not complete the study due to cognitive decline, therefore, slightly skewing results towards participants capable of adhering to the protocols and follow-up.

\section{Future directions}

Future large RCT aiming to determine the effectiveness of dietary supplements and nutraceuticals to improve cognition in the elderly should include comprehensive neuropsychological testing. Although general screening measures may be optimal for physicians, to detect subtle changes across cognitive domains requires sensitive testing that may take from $2 \mathrm{~h}$ to $2 \mathrm{~d}$ to administer. This degree of assessment provides challenges, as more than just screening tests for dementia are needed to measure cognitive outcomes. A focus on the overall dietary pattern has been proposed as an intervention strategy to prevent cognitive decline, yet the current evidence from RCT is relatively weak overall ${ }^{(17)}$. However, dietary supplements use is associated with greater compliance to healthy dietary and lifestyle habits ${ }^{(100)}$. Therefore, the combination of dietary and nutraceutical interventions may be a promising strategy to improve cognitive function. Advances in molecular nutrition, nutrigenetics and nutrigenomics must be considered in designing future studies investigating dietary supplements and nutraceuticals $^{(101)}$, particularly regarding APOE4 genotyping of participants in studies of cognitive function. The consideration of several blood-based biomarkers, such as $n-3$ fatty acid status $^{(36)}$, will be important in future trials. It also remains to be seen whether mandatory fortification measures, such as folic acid, will result in long-term cognitive benefits ${ }^{(102)}$. Curcumin is a botanical of current interest, proposed to improve cognition, but to date, mixed results have been reported ${ }^{(38,103)}$. We suggest consideration of less-known botanicals, such as Yamabushitake $^{(104)}$, which has shown benefit in a short-term trial. The absorption and bioavailability of such botanicals both in the gastrointestinal tract and across the blood-brain barrier must also be considered before long-term trials commence. Lastly, interest in nootropic supplements is also increasing, with shortterm benefits in young healthy individuals ${ }^{(105)}$ but the potential of these supplements to improve cognition in the elderly is worthy of future research.

\section{Conclusion}

The popularity of dietary supplements and nutraceuticals continues to increase; however, this systematic review has failed to locate sufficient evidence to justify long-term use to improve cognitive function in elderly individuals aged over 65 years. However, there is limited evidence regarding the interaction of blood biomarkers such as $n-3$ fatty acid status and genetic risk factors including APOE4, which suggests the potential benefits of nutraceuticals may be dependent on multiple factors. Until rigorous long-term clinical trials are conducted, claims made by manufacturers and under qualified health advisors, must be interpreted with extreme caution as to avoid potential side effects and future health complications. Advances in molecular nutrition and nutrigenomics will continue to uncover novel compounds, and may allow for re-investigation of well-known nutraceuticals.
These findings may result in benefit to the elderly, especially those suffering from impaired cognition. This review suggests that future long-term clinical trials utilise sensitive neuropsychological testing of participants when consuming nutraceuticals, and the recruitment of individuals at high risk of developing MCI or dementia, to determine if benefits exist to improving cognitive function or delaying cognitive decline.

\section{Acknowledgements}

This study was supported by the University of Canberra Health Research Institute - Research and Support Development Program.

J. K. and N. N. designed the study and devised the research questions, N. N., N. M. D. C, E. N. G. and L. D. carried out the literature review and data analysis. N. M. D. C. wrote the manuscript with contributions from D. B. P., J. T., A. J. M. K., D. D. M. and N. N. All authors read and contributed to the finalisation of the manuscript.

The authors declare that there are no conflicts of interest.

\section{References}

1. He W, Goodkind D \& Kowal P (2016) An Aging World: 2015. International Population Reports. Washington, DC: U.S. Census Bureau.

2. Wasay M, Grisold W, Carroll W, et al. (2016) World Brain Day 2016: celebrating brain health in an ageing population. Lancet Neurol 15, 1008.

3. Ngandu T, Lehtisalo J, Solomon A, et al. (2015) A 2 year multidomain intervention of diet, exercise, cognitive training, and vascular risk monitoring versus control to prevent cognitive decline in at-risk elderly people (FINGER): a randomised controlled trial. Lancet 385, 2255-2263.

4. Rountree SD, Chan W, Pavlik VN, et al. (2009) Persistent treatment with cholinesterase inhibitors and/or memantine slows clinical progression of Alzheimer disease. Alzheimer's Res Ther 1, 7 .

5. Meguro M, Kasai M, Akanuma K, et al. (2008) Comprehensive approach of donepezil and psychosocial interventions on cognitive function and quality of life for Alzheimer's disease: the Osaki-Tajiri Project. Age Ageing 37, 469-473.

6. Takizawa C, Thompson PL, van Walsem A, et al. (2015) Epidemiological and economic burden of Alzheimer's disease: a systematic literature review of data across Europe and the United States of America. I Alzheimer's Dis $\mathbf{4 3}$, 1271-1284.

7. Siddiqui MJ, Min CS, Verma RK, et al. (2014) Role of complementary and alternative medicine in geriatric care: a mini review. Pharmacog Rev 8, 81-87.

8. Harrington CR (2012) The molecular pathology of Alzheimer's disease. Neuroimaging Clin N Am 22, 11-22, vii.

9. Reitz C (2012) Alzheimer's disease and the amyloid cascade hypothesis: a critical review. Int J Alzheimers Dis 2012, 11.

10. Willette AA, Bendlin BB, Starks EJ, et al. (2015) Association of insulin resistance with Cerebral glucose uptake in late middle-aged adults at risk for Alzheimer disease. JAMA Neurol 72, 1013-1020.

11. Naeini AM, Elmadfa I, Djazayery A, et al. (2014) The effect of antioxidant vitamins $\mathrm{E}$ and $\mathrm{C}$ on cognitive performance of the elderly with mild cognitive impairment in Isfahan, Iran: a double-blind, randomized, placebo-controlled trial. Eur $J$ Nutr 53, 1255-1262. 
12. Opara JA (2012) Activities of daily living and quality of life in Alzheimer disease. J Med Life 5, 162-167.

13. Djernes JK (2006) Prevalence and predictors of depression in populations of elderly: a review. Acta Psychiatr Scand 113, 372-387.

14. Sando SB, Melquist S, Cannon A, et al. (2008) APOE $\varepsilon 4$ lowers age at onset and is a high risk factor for Alzheimer's disease; a case control study from central Norway. BMC Neurol 8, 9.

15. Xu W, Tan L, Wang H-F, et al. (2015) Meta-analysis of modifiable risk factors for Alzheimer's disease. J Neurol Neurosurg Psychiatry 86, 1299.

16. van de Rest O, Berendsen AAM, Haveman-Nies A, et al. (2015) Dietary patterns, cognitive decline, and dementia: a systematic review. Adv Nutr 6, 154-168.

17. Canevelli M, Lucchini F, Quarata F, et al. (2016) Nutrition and dementia: evidence for preventive approaches? Nutrients $\mathbf{8}, 144$.

18. Mogendi JB, De Steur H, Gellynck X, et al. (2016) Consumer evaluation of food with nutritional benefits: a systematic review and narrative synthesis. Int J Food Sci Nutr 67, 355-371.

19. Qato DM, Wilder J, Schumm LP, et al. (2016) Changes in prescription and over-the-counter medication and dietary supplement use among older adults in the United States, 2005 vs 2011. JAMA Intern Med 176, 473-482.

20. Gahche J, Bailey R, Burt V, et al. (2011) Dietary supplement use among U.S. adults has increased since NHANES III (1988-1994). NCHS Data Brief, 1-8.

21. Petroczi A, Taylor G \& Naughton DP (2011) Mission impossible? Regulatory and enforcement issues to ensure safety of dietary supplements. Food Chem Toxicol 49, 393-402.

22. Gabriels G \& Lambert M (2013) Nutritional supplement products: does the label information influence purchasing decisions for the physically active? Nutr J 12, 133.

23. Heuberger R (2012) Polypharmacy and food-drug interactions among older persons: a review. J Nutr Gerontol Geriatr 31, 325-403.

24. Deldicque L \& Francaux M (2016) Potential harmful effects of dietary supplements in sports medicine. Curr Opin Clin Nutr Metab Care 19, 439-445.

25. Geller AI, Shehab N, Weidle NJ, et al. (2015) Emergency department visits for adverse events related to dietary supplements. $N$ Engl J Med 373, 1531-1540.

26. Mursu J, Robien K, Harnack LJ, et al. (2011) Dietary supplements and mortality rate in older women: the Iowa Women's Health Study. Arch Intern Med 171, 1625-1633.

27. Das L, Bhaumik E, Raychaudhuri U, et al. (2012) Role of nutraceuticals in human health. J Food Sci Technol 49, 173-183.

28. Nasri H, Baradaran A, Shirzad H, et al. (2014) New concepts in nutraceuticals as alternative for pharmaceuticals. Int J Prev Med 5, 1487-1499.

29. Kalra EK (2003) Nutraceutical-definition and introduction. AAPS PharmSci 5, 27-28.

30. Geleijnse JM, Giltay EJ \& Kromhout D (2012) Effects of $n-3$ fatty acids on cognitive decline: a randomized, double-blind, placebo-controlled trial in stable myocardial infarction patients. Alzheimers Dement 8, 278-287.

31. McDougall GJ, Austin-Wells V \& Zimmerman T (2005) Utility of nutraceutical products marketed for cognitive and memory enhancement. J Holist Nurs 23, 415-433.

32. Alzheimer's Association (2017) Alternative treatments. http:// www.alz.org/alzheimers_disease_alternative_treatments.asp (accessed February 2017).
33. Zandi PP, Anthony JC, Khachaturian AS, et al. (2004) Reduced risk of Alzheimer disease in users of antioxidant vitamin supplements: the Cache County Study. Arch Neurol 61, 82-88.

34. Moher D, Liberati A, Tetzlaff J, et al. (2009) Preferred reporting items for systematic reviews and meta-analyses: the PRISMA statement. PLoS Med 6, e1000097.

35. Clarke R, Bennett D, Parish S, et al. (2014) Effects of homocysteine lowering with $\mathrm{B}$ vitamins on cognitive aging: meta-analysis of 11 trials with cognitive data on 22,000 individuals. Am J Clin Nutr 100, 657-666.

36. Oulhaj A, Jernerén F, Refsum H, et al. (2016) Omega-3 fatty acid status enhances the prevention of cognitive decline by b vitamins in mild cognitive impairment. $J$ Alzheimers Dis 50, 547-557.

37. Ford AH, Flicker L, Alfonso $\mathrm{H}$, et al. (2010) Vitamins $\mathrm{B}_{12}, \mathrm{~B}_{6}$, and folic acid for cognition in older men. Neurology $\mathbf{7 5}$, 1540-1547.

38. Rainey-Smith SR, Brown BM, Sohrabi HR, et al. (2016) Curcumin and cognition: a randomised, placebo-controlled, double-blind study of community-dwelling older adults. Br J Nutr 115, 2106-2113.

39. Higgins JP \& Green S (2011) Cochrane Handbook for Systematic Reviews of Interventions, vol. 4. Chichester: John Wiley \& Sons.

40. Chew EY, Clemons TE, Agron E, et al. (2015) Effect of omega-3 fatty acids, lutein/zeaxanthin, or other nutrient supplementation on cognitive function: the AREDS2 randomized clinical trial. JAMA 314, 791-801.

41. Grodstein F, O'Brien J, Kang JH, et al. (2013) Long-term multivitamin supplementation and cognitive function in men: a randomized trial. Ann Intern Med 159, 806-+

42. McNeill G, Avenell A, Campbell MK, et al. (2007) Effect of multivitamin and multimineral supplementation on cognitive function in men and women aged 65 years and over: a randomised controlled trial. Nutr J 6, 10.

43. Rossom RC, Espeland MA, Manson JE, et al. (2012) Calcium and vitamin D supplementation and cognitive impairment in the Women's Health Initiative. I Am Geriatr $S o c \mathbf{6 0}$, 2197-2205.

44. Aisen PS, Schneider LS, Sano M, et al. (2008) High-dose B vitamin supplementation and cognitive decline in Alzheimer disease: a randomized controlled trial. JAMA $\mathbf{3 0 0}$, $1774-1783$

45. Dangour AD, Allen E, Clarke R, et al. (2015) Effects of vitamin B-12 supplementation on neurologic and cognitive function in older people: a randomized controlled trial. $A m J$ Clin Nutr 102, 639-647.

46. de Jager CA, Oulhaj A, Jacoby R, et al. (2012) Cognitive and clinical outcomes of homocysteine-lowering B-vitamin treatment in mild cognitive impairment: a randomized controlled trial. Int J Geriatr Psychiatry 27, 592-600.

47. Kwok T, Lee J, Law CB, et al. (2011) A randomized placebo controlled trial of homocysteine lowering to reduce cognitive decline in older demented people. Clin Nutr $\mathbf{3 0}$, 297-302.

48. McMahon JA, Green TJ, Skeaff CM, et al. (2006) A controlled trial of homocysteine lowering and cognitive performance. $N$ Engl J Med 354, 2764-2772.

49. Van Der Zwaluw NL, Dhonukshe-Rutten RAM, Van Wijngaarden JPV, et al. (2014) Results of 2-year vitamin $\mathrm{B}$ treatment on cognitive performance; secondary data from an RCT. Neurology 83, 2158-2166.

50. Walker JG, Batterham PJ, Mackinnon AJ, et al. (2012) Oral folic acid and vitamin B-12 supplementation to prevent cognitive decline in community-dwelling older adults with 
depressive symptoms - the Beyond Ageing Project: a randomized controlled trial. Am J Clin Nutr 95, 194-203 [corrected] [published erratum appears in Am J Clin Nutr 2012 Aug;96(2):448].

51. Dangour AD, Allen E, Elbourne D, et al. (2010) Effect of 2-y $n$-3 long-chain polyunsaturated fatty acid supplementation on cognitive function in older people: a randomized, double-blind, controlled trial. Am J Clin Nutr 91, 1725-1732.

52. Quinn J, Raman R, Thomas R, et al. (2010) Docosahexaenoic acid supplementation and cognitive decline in Alzheimer disease: a randomized trial. JAMA 304, 1903-1911.

53. DeKosky ST, Williamson JD, Fitzpatrick AL, et al. (2008) Ginkgo biloba for prevention of dementia: a randomized controlled trial. JAMA 300, 2253-2262.

54. Snitz BE, O'Meara ES, Carlson MC, et al. (2009) Ginkgo biloba for preventing cognitive decline in older adults: a randomized trial. JAMA 302, 2663-2670.

55. Dodge HH, Zitzelberger T, Oken BS, et al. (2008) A randomized placebo-controlled trial of Ginkgo biloba for the prevention of cognitive decline. Neurology 70, 1809-1817.

56. Grodstein F, Kang JH, Glynn RJ, et al. (2007) A randomized trial of beta carotene supplementation and cognitive function in men - the Physicians' Health Study II. Arch Intern Med 167, 2184-2190.

57. $\mathrm{Hu}$ K, Riemersma-van der Lek RF, Patxot M, et al. (2016) Progression of dementia assessed by temporal correlations of physical activity: results from a 3.5-year, longitudinal randomized controlled trial. Sci Rep 6, 27742.

58. Turner RS, Thomas RG, Craft S, et al. (2015) A randomized, double-blind, placebo-controlled trial of resveratrol for Alzheimer disease. Neurology 85, 1383-1391.

59. Riemersma-van der Lek RF, Swaab DF, Twisk J, et al. (2008) Effect of bright light and melatonin on cognitive and noncognitive function in elderly residents of group care facilities: a randomized controlled trial. JAMA 299, 2642-2655.

60. Vellas B, Coley N, Ousset PJ, et al. (2012) Long-term use of standardised Ginkgo biloba extract for the prevention of Alzheimer's disease (GuidAge): a randomised placebocontrolled trial. Lancet Neurol 11, 851-859.

61. Veronese N, Stubbs B, Maggi S, et al. (2017) Low-dose aspirin use and cognitive function in older age: a systematic review and meta-analysis. J Am Geriatr Soc 65, 1763-1768.

62. Yetley EA (2007) Multivitamin and multimineral dietary supplements: definitions, characterization, bioavailability, and drug interactions. Am J Clin Nutr 85, 269S-276S.

63. Scragg R, Stewart AW, Waayer D, et al. (2017) Effect of monthly high-dose vitamin d supplementation on cardiovascular disease in the vitamin $\mathrm{d}$ assessment study: a randomized clinical trial. JAMA Cardiol 2, 608-616.

64. Smith AD, Smith SM, de Jager CA, et al. (2010) Homocysteine-lowering by $\mathrm{B}$ vitamins slows the rate of accelerated brain atrophy in mild cognitive impairment: a randomized controlled trial. PLOS ONE 5, e12244.

65. Kennedy OD (2016) B vitamins and the brain: mechanisms, dose and efficacy - a review. Nutrients $\mathbf{8}, 68$.

66. Yassine HN, Rawat V, Mack WJ, et al. (2016) The effect of APOE genotype on the delivery of DHA to cerebrospinal fluid in Alzheimer's disease. Alzheimers Res Ther 8, 25.

67. Panagiotakos D (2014) A Mediterranean diet supplemented with olive oil or nuts reduces the incidence of major cardiovascular events in high-risk patients. BMJ Evid Based Med 19, 10.

68. Martinez-Lapiscina EH, Clavero P, Toledo E, et al. (2013) Virgin olive oil supplementation and long-term cognition: the Predimed-Navarra randomized, trial. J Nutr Health Aging 17, 544-552.
69. Freund-Levi Y, Eriksdotter-Jonhagen M, Cederholm T, et al. (2006) Omega-3 fatty acid treatment in 174 patients with mild to moderate Alzheimer disease: OmegAD study: a randomized double-blind trial. Arch Neurol 63, 1402-1408.

70. Freund-Levi Y, Basun H, Cederholm T, et al. (2008) Omega3 supplementation in mild to moderate Alzheimer's disease: effects on neuropsychiatric symptoms. Int $J$ Geriatr Psychiatry 23, 161-169.

71. Ketz JS, Rodavich MC \& Barnes KM (2013) Absorption of marine vs non-marine sources of EPA and DHA. FASEB J $\mathbf{2 7}$, 867.863 .

72. Therapeutic Goods Administration (2009) Australian public assessment report for melatonin. Proprietary Product Name: Circadin. https://www.tga.gov.au/sites/default/files/ausparcircadin.pdf (accessed September 2017).

73. Nestor SM, Rupsingh R, Borrie M, et al. (2008) Ventricular enlargement as a possible measure of Alzheimer's disease progression validated using the Alzheimer's disease neuroimaging initiative database. Brain 131, 2443-2454.

74. Weinmann S, Roll S, Schwarzbach C, et al. (2010) Effects of Ginkgo biloba in dementia: systematic review and metaanalysis. BMC Geriatr 10, 14.

75. Byers T (1999) What can randomized controlled trials tell us about nutrition and cancer prevention? CA Cancer J Clin $\mathbf{4 9}$, 353-361.

76. Wang H, Naghavi M, Allen C, et al. (2016) Global, regional, and national life expectancy, all-cause mortality, and causespecific mortality for 249 causes of death, 1980-2015: a systematic analysis for the Global Burden of Disease Study 2015. Lancet 388, 1459-1544.

77. Durga J, van Boxtel MP, Schouten EG, et al. (2007) Effect of 3 -year folic acid supplementation on cognitive function in older adults in the FACIT trial: a randomised, double blind, controlled trial. Lancet 369, 208-216.

78. Rundblad A, Holven KB, Ottestad I, et al. (2017) High-quality fish oil has a more favourable effect than oxidised fish oil on intermediate-density lipoprotein and LDL subclasses: a randomised controlled trial. Br J Nutr, 117 1291-1298.

79. Burton GW, Traber MG, Acuff RV, et al. (1998) Human plasma and tissue alpha-tocopherol concentrations in response to supplementation with deuterated natural and synthetic vitamin E. Am J Clin Nutr 67, 669-684.

80. Lucock M \& Yates Z (2005) Folic acid - vitamin and panacea or genetic time bomb? Nat Rev Genet 6, 235-240.

81. Moore EM, Ames D, Mander AG, et al. (2014) Among vitamin B12 deficient older people, high folate levels are associated with worse cognitive function: combined data from three cohorts. J Alzheimers Dis 39, 661-668.

82. Schiepers OJG, Harris SE, Gow AJ, et al. (2012) APOE E4 status predicts age-related cognitive decline in the ninth decade: longitudinal follow-up of the Lothian Birth Cohort 1921. Mol Psychiatry 17, 315-324.

83. Liu C-C, Kanekiyo T, Xu H, et al. (2013) Apolipoprotein E and Alzheimer disease: risk, mechanisms, and therapy. Nat Rev Neurol 9, 106-118.

84. B RC, Hare DJ, Lind M, et al. (2017) The APOE epsilon4 Allele Is Associated with Lower Selenium Levels in the Brain: Implications for Alzheimer's Disease. ACS Chemical Neurosci 8, 1459-1464.

85. Minihane AM (2016) Impact of genotype on EPA and DHA status and responsiveness to increased intakes. Nutrients $\mathbf{8}, 123$.

86. Lucock MD, Martin CE, Yates ZR, et al. (2014) Diet and our genetic legacy in the recent anthropocene: a Darwinian perspective to nutritional health. I Evid Based Complementary Altern Med 19, 68-83. 
87. Meyer BJ, Onyiaodike CC, Brown EA, et al. (2016) Maternal plasma DHA levels increase prior to 29 days post-LH surge in women undergoing frozen embryo transfer: a prospective, observational study of human pregnancy. $J$ Clin Endocrinol Metab 101, 1745-1753.

88. Farrer LA, Cupples LA, Haines JL, et al. (1997) Effects of age, sex, and ethnicity on the association between apolipoprotein $\mathrm{E}$ genotype and Alzheimer disease. A meta-analysis. APOE and Alzheimer Disease Meta Analysis Consortium. JAMA 278, 1349-1356.

89. Hathcock J (2001) Dietary supplements: how they are used and regulated. J Nutr 131, 1114s-1117s.

90. Guallar E, Stranges S, Mulrow C, et al. (2013) Enough is enough: Stop wasting money on vitamin and mineral supplements. Ann Intern Med 159, 850-851.

91. Bjelakovic G, Nikolova D \& Gluud C (2013) Antioxidant supplements to prevent mortality. JAMA 310, 1178-1179.

92. Abner EL, Schmitt FA, Mendiondo MS, et al. (2011) Vitamin E and all-cause mortality: a meta-analysis. Curr Aging Sci $\mathbf{4}$, $158-170$

93. Macpherson H, Pipingas A \& Pase MP (2013) Multivitaminmultimineral supplementation and mortality: a meta-analysis of randomized controlled trials. Am J Clin Nutr 97, 437-444.

94. Li K, Kaaks R, Linseisen J, et al. (2012) Vitamin/mineral supplementation and cancer, cardiovascular, and all-cause mortality in a German prospective cohort (EPIC-Heidelberg). Eur J Nutr 51, 407-413.

95. Fortmann SP, Burda BU, Senger CA, et al. (2013) Vitamin and mineral supplements in the primary prevention of cardiovascular disease and cancer: an updated systematic evidence review for the U.S. preventive services task force. Ann Intern Med 159, 824-834.

96. Onakpoya IJ \& Heneghan CJ (2017) The efficacy of supplementation with the novel medical food, Souvenaid, in patients with Alzheimer's disease: a systematic review and meta-analysis of randomized clinical trials. Nutr Neurosci $\mathbf{2 0}$, 219-227.
97. Soininen H, Visser P, Kivipelto M, et al. (2016) A clinical trial investigating the effects of fortasyn connect (Souvenaid) in prodromal Alzheimer's disease: results of the LipiDiDiet study. Neurobiol Aging 39, S23.

98. Andrieu S, Guyonnet S, Coley N, et al. (2017) Effect of longterm omega 3 polyunsaturated fatty acid supplementation with or without multidomain intervention on cognitive function in elderly adults with memory complaints (MAPT): a randomised, placebo-controlled trial. Lancet Neurol $\mathbf{1 6}$, 377-389.

99. Yassine HN \& Schneider LS (2017) Lessons from the Multidomain Alzheimer Preventive Trial. Lancet Neurol 16, 585-586.

100. Pouchieu C, Andreeva VA, Peneau S, et al. (2013) Sociodemographic, lifestyle and dietary correlates of dietary supplement use in a large sample of French adults: results from the NutriNet-Sante cohort study. Br J Nutr 110, 1480-1491.

101. Schwartz B (2014) New criteria for supplementation of selected micronutrients in the era of nutrigenetics and nutrigenomics. Int J Food Sci Nutr 65, 529-538.

102. Beckett EL, Martin C, Boyd L, et al. (2017) Reduced plasma homocysteine levels in elderly Australians following mandatory folic acid fortification; a comparison of two crosssectional cohorts. J Nutr Intermed Metab 8, 14-20.

103. Baum L, Lam CW, Cheung SK, et al. (2008) Six-month randomized, placebo-controlled, double-blind, pilot clinical trial of curcumin in patients with Alzheimer disease. J Clin Psychopharmacol 28, 110-113.

104. Mori K, Inatomi S, Ouchi K, et al. (2009) Improving effects of the mushroom Yamabushitake (Hericium erinaceus) on mild cognitive impairment: a double-blind placebo-controlled clinical trial. Phytother Res 23, 367-372.

105. Solomon TM, Leech J, deBros GB, et al. (2016) A randomized, double-blind, placebo controlled, parallel group, efficacy study of alpha BRAIN(R) administered orally. Hum Psychopharmacol 31, 135-143. 